\title{
The Worst Drug Rule Revisited: Mathematical Modeling of Cyclic Cancer Treatments
}

\author{
Allen A. Katouli • Natalia L. Komarova
}

Received: 16 October 2009 / Accepted: 23 March 2010 / Published online: 16 April 2010

(C) The Author(s) 2010. This article is published with open access at Springerlink.com

\begin{abstract}
In drug treatments of cancer, cyclic treatment strategies are characterized by alternating applications of two (or more) different drugs, given one at a time. One of the main problems of drug treatment in cancer is associated with the generation of drug resistance by mutations of cancerous cells. We use mathematical methods to develop general guidelines on optimal cyclic treatment scheduling, with the aim of minimizing the resistance generation. We define a condition on the drugs' potencies which allows for a relatively successful application of cyclic therapies. We find that the best strategy is to start with the stronger drug, but use longer cycle durations for the weaker drug. We further investigate the situation where a degree of crossresistance is present, such that certain mutations cause cells to become resistant to both drugs simultaneously. We show that the general rule (best-drug-first, worst-druglonger) is unchanged by the presence of cross-resistance. We design a systematic method to test all strategies and come up with the optimal timing and drug order. The role of various constraints in the optimal therapy design, and in particular, suboptimal treatment durations and drug toxicity, is considered. The connection with the "worst drug rule" of Day (Cancer Res. 46:3876, 1986b) is discussed.
\end{abstract}

Keywords Stochastic modeling of cancer $\cdot$ CML

\section{Introduction}

Mathematical modeling of cancer therapy performed by Goldie and Coldman (1979, 1983b, 1998), Goldie et al. (1982), Coldman and Goldie (1985) and Day (1986b) in the late 1970s and the 1980s had a widespread impact on the design of new chemotherapy regimens for testing in clinical trials (Norton and Day 1985). This was

\footnotetext{
A.A. Katouli · N.L. Komarova ( $\square)$

Department of Mathematics, University of California Irvine, Irvine, CA 92697, USA

e-mail: komarova@uci.edu
} 
an exciting time in the history of mathematical modeling in medicine. Stochastic models applied to generation of drug resistance and treatment optimization have been used in scheduling chemotherapy treatments. In particular, wet lab oncologists undertook the task of testing a specific, purely theoretical rule proposed by Day (1986b): the "worst drug rule."

The worst drug rule is a general optimization principle which states that if two non-cross-resistant drugs are used intermittently in treatment, then the drug with the weaker killing rate has to be applied first and/or for a longer period of time. This rule has been derived computationally, in the context of non-cross-resistant drugs, by considering 16 different treatment strategies listed below:

$\begin{array}{llll}A A A A A A A A A A A A & B B B A A A A A A A A A & B A B A B A B A B A B A & A B B B A B B B A B B B \\ A A A A A A A A B B B & A A A A A A B B B B B & B B B A A A B B B A A A & B B B A B B B A B B A \\ A A A B A A A A A A B & A A A B B B A A B B B & B B B B B B A A A A A & B B B B B B B B A A A \\ B A A A B A A A B A A A & A B A B A B A B A B A B & A A A B B B B B B B B & B B B B B B B B B B B\end{array}$

Here, each letter " $A$ " or " $B$ " denotes a single course of treatment with drug $A$ or $B$ respectively; a course is assumed to last one month. Each treatment strategy consists of the total of 12 courses (which fixes the length of treatment). The parameters were chosen such that the total length of treatment is sufficient to eradicate all the susceptible mutants. For each of the treatment strategies, the probability of treatment success was calculated for different coefficient values, using the methodology developed by Day (1986a). In particular, the mutation rates, the killing rates and the proliferation rates were varied. The conclusion of the study was that, with a few exceptions, the most successful strategies are the ones where the "worst" drug (that is, the drug with a weaker killing rate) was applied earlier, or for a longer duration, compared to the better drug. The authors distinguish two variants of the worst drug rule (Norton and Day 1985): (a) "use more of the worst drug" and (b) "use the worst drug early."

In this paper, we pose a question similar to that raised by Day (1986b): What is the optimal timing of treatment in the context of cyclic therapy? Our analysis employs a wider variety of methods and is more general and systematic. In particular, instead of testing a subset of 16 treatment strategies, we test all cyclic treatment strategies to find the optimal cycle duration and the drug sequence. In addition, we extend the studies to drugs with cross-resistance.

We distinguish two different characteristics of the drugs: their potency and their activity spectrum. By potency, we mean how effectively a drug kills cells that are susceptible to the drug; this is similar to the killing rate discussed by Day (1986b). We will say that a drug is characterized by a broad activity spectrum if it is effective against a large spectrum of mutant cells. On the other hand, a drug with a narrow activity spectrum is a more specific agent, which is active against a relatively small number of cell variants.

The main findings of this paper are the following:

- In order for a cyclic treatment to be effective, the drugs' potencies must satisfy a certain condition (condition (33) in the paper), which we call the condition of "mutual strength." For realistic parameters, drugs which are not mutually strong will yield very poor probabilities of treatment success, if applied cyclically.

- The general rule for cyclic treatments with mutually strong drugs of similar activity spectra is: use the best drug first, but use the worst drug for longer. 
- This "best drug first" rule becomes more important if the treatment time is suboptimal.

- The presence of cross-resistance does not change this rule.

- Toxicity considerations can change this rule, in particular, when the worst drug is more toxic.

- The general rule for cyclic treatments with mutually strong drugs of similar potency and different activity spectra is: use the less active drug first, and use the more active drug for longer. This rule only applies for short-term treatments.

- The optimal cycle durations for given parameters such as drug strengths and the mutations rates, can be calculated, and do not depend on the tumor size. They do depend on other parameters, and in particular, on the total treatment duration.

In this paper, we use stochastic and deterministic modeling of cancer growth and treatment. The last four decades are characterized by significant developments in the field of anticancer therapy modeling, see e.g. the following recent reviews: Sanga et al. (2006), Byrne et al. (2006); Bellomo et al. (2008a, 2008b); Swierniak et al. (2009); Deisboeck et al. (2009). Cancer therapy can be modeled on several spatial scales. Molecular processes, such as mutations or chromosomal changes, happen on the subcellular scale. They are responsible for malignant transformations that turn a healthy cell into a malignant one. Intracellular signaling and drug-cell interactions are also described on the subcellular scale. Cells in an organ interact by means of direct cell-to-cell signaling as well as through the process of selection; they compete for space and nutrients. The action of the drugs influences the population dynamics of cells. The resulting dynamics happen on the cellular level. The macroscopic description, or the organismal scale, is appropriate when studying a larger population of cancerous cells, which can be viewed as a continuum. The largest scale is the inter-organismal, or population scale. There, the epidemiology and statistics of cancer, and in particular, the population-level effects of various cancer therapies, are studied. Some modelers restrict their consideration to a particular scale, while others use multi-scale modeling, see e.g. Preziosi (2003).

In this paper, we concentrate on an intermediate, cellular scale of modeling, and use both stochastic and deterministic methodologies to study the effects of cyclic therapy on cancer. For the existing literature on this type of modeling, see e.g. Wodarz and Komarova (2005).

The rest of the paper is organized as follows. In Sect. 2 we outline the stochastic model of cyclic drug treatment in the presence of cross-resistance. In Sect. 3 we design a simple deterministic model capable of describing the case of mutually strong drugs, both non-cross-resistant and cross-resistant. In Sect. 4 we present a numerical analysis of probabilities of treatment success for various drug potencies and show that mutually strong drugs yield the best probabilities of treatment success. Section 5 first presents an analysis for mutually strong drugs of different potencies and equal activity spectra, and subsequently handles the drugs of equal potencies and different activity spectra. Section 6 extends our considerations to short treatments. Section 7 addresses the problem of toxic drugs and shows how our methods apply to such situations. Section 8 contains discussion and conclusions. 


\section{Stochastic Modeling for Cyclic Treatments}

\subsection{Review of the General Framework}

The basic mathematical framework described here was developed by Komarova and Wodarz (2005); Komarova (2006) and goes back to early works of Goldie and Coldman (1979, 1983a, 1983b, 1998); Coldman and Goldie $(1985,1986)$. We denote by $i_{s}$ the number of cells of resistance class $s$. Here, $s$ is a binary number that goes from 0 to $n=2^{m}$, where $m$ is the number of different drugs used. In this paper we deal with two-drug treatments, therefore we set $m=2$, which leads to the existence of four separate resistance classes, see Fig. 1(a): $s=00$, fully susceptible; $s=10$, resistant to drug 1 and susceptible to drug 2; $s=01$, resistant to drug 2 and susceptible to drug 1; and $s=11$, resistant to both drugs (or, fully-resistant). Let $\varphi_{i_{0}, \ldots, i_{n}}(t)$ denote the probability that at time $t$ there are $i_{s}$ cells of resistance class $s$, for all classes $s$. Suppose that cancerous cells divide with rate $l_{s}$, and die with rate $D_{s}$. Coefficients can depend on the treatment protocol. In particular, the death rate of cells, $D_{s}$, is comprised of the "natural" rate of cell death in an untreated tumor and the action of the $\operatorname{drug}(\mathrm{s})$, if any, upon the cells. Therefore, in general, this quantity is a function of time.

The mutation rates are defined for all edges of the diagrams in Fig. 1 and denoted by $u$ with the corresponding indices. In particular, in Fig. 1, $u_{1}$ denotes the rate at which mutations resistant to drug 1 (and not to drug 2) are generated. Similarly, the rate $u_{2}$ describes the generation of mutations conferring resistance to drug 2 only. The possibility of mutations conferring resistance to both drugs simultaneously (the so-called cross-resistance) is captured by the rate $u_{12}$; in the absence of cross-resistance, $u_{12}=0$. The mathematical formalism for studying cross-resistance in cancer drug treatments was introduced recently by Komarova et al. (2009); some additional details on the modeling can be found in Katouli (2009).

We assume that the timing of separate kinetic events is exponentially distributed, and construct a linear birth-death process (for a review of biological applications of the theory of birth-death processes, see Novozhilov et al. (2006)). Its Kolmogorov forward equation can be written as

$$
\dot{\varphi}_{i_{0}, \ldots, i_{n}}=\sum_{s=0}^{n} Q^{s},
$$

where $Q^{s}$ is the contribution obtained from considering probabilities of reproduction and death of resistance type $s$,

$$
\begin{aligned}
Q^{s}= & \varphi_{i_{0} \ldots i_{s}-1 \ldots i_{n}}\left(i_{s}-1\right) l_{s}\left(1-\sum_{j} u_{s \rightarrow j}\right)+i_{s} l_{s} \sum_{j} \varphi_{i_{0} \ldots i_{s} \ldots i_{j}-1 \ldots u_{s \rightarrow j}} \\
& +\varphi_{i_{0} \ldots i_{s}+1 \ldots i_{n}}\left(i_{s}+1\right) D_{s}-\varphi_{i_{0} \ldots i_{n}} i_{s}\left(l_{s}+D_{s}\right)
\end{aligned}
$$

here, symbols $u_{s \rightarrow j}$ denote the mutation rates for all arrows originating at $s$. The first group of terms above represents cell divisions of class $s$ without mutations, the second group is all cell reproductions of class $s$ with mutations, the third term has 
(a) Treatment with drug 1:

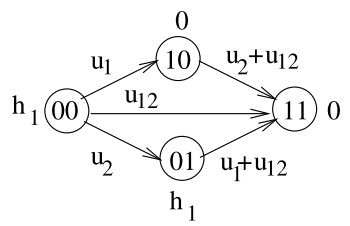

Treatment with drug 2:

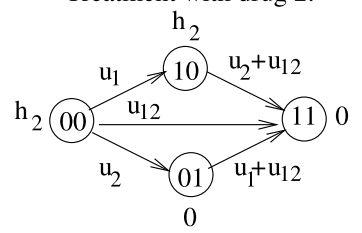

(b)

\begin{tabular}{|c|c|c|c|c|c|c|}
\hline No treatment & Drug 1 & Drug 2 & Drug 1 & Drug 2 & Drug 1 & Drug \\
\hline $\mathrm{t}_{*}$ & $\Delta \mathrm{t}_{1}$ & $\Delta \mathrm{t}_{2}$ & $\Delta \mathrm{t}_{1}$ & $\Delta \mathrm{t}_{2}$ & $\Delta \mathrm{t}_{1}$ & \\
\hline
\end{tabular}

(c) The averaged treatment:

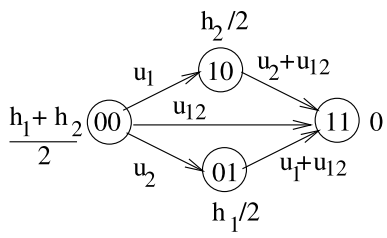

Fig. 1 Cyclic two-drug treatments. (a) The mutation diagrams and drug-induced death rates for treatments with drug 1 and drug 2 . The resistance types are represented by circles with binary indices; the drug-induced death rates are marked next to the circles; the mutation rates are indicated next to the arrows. (b) A cyclic treatment protocol. (c) The average combination treatment

the meaning of cell death in class $s$, and the last term is "no change." The probability generating function can be defined in a usual way (Gardiner 2004):

$$
\Psi(\vec{\xi} ; t)=\sum_{s=0}^{n} \varphi_{i_{0}, \ldots, i_{n}}(t) \xi_{0}^{i_{0}} \ldots \xi_{n}^{i_{n}}
$$

with $\vec{\xi}=\left(\xi_{0}, \ldots, \xi_{n}\right)$. The above transformation maps an infinite number of unknown functions, $\varphi_{i_{0}, \ldots, i_{n}}(t)$, onto one function of a continuous variable, $\Psi(\vec{\xi} ; t)$. This function satisfies the partial differential equation, derived by standard methods (see e.g. Bailey 1964):

$$
\frac{\partial \Psi}{\partial t}=\sum_{s=0}^{n} \frac{\partial \Psi}{\partial \xi_{s}}\left(l_{s}\left(1-\sum_{j} u_{s \rightarrow j}^{\text {out }}\right) \xi_{s}^{2}+\left[\sum_{j} l_{s} u_{s \rightarrow j}^{\text {out }} \xi_{j}^{\text {out }}-\left(l_{s}+D_{s}\right)\right] \xi_{s}+D_{s}\right) .
$$

To obtain the above equation, the master equation (1) is multiplied by $\xi_{0}^{i_{0}} \ldots \xi_{n}^{i_{n}}$ and summed over all the indices; the terms containing $i_{s}$ can then be rewritten as partial derivatives of $\Psi$ with respect to $\xi_{s}$ (Komarova 2006). The coefficients, such as $D_{s}$, on the right-hand side can be functions of time.

Following the standard technique for hyperbolic equations, we have the following system of equations for characteristics:

$$
\dot{\xi}_{s}=-\mathcal{F}_{s}(\vec{\xi} ; t),
$$


where

$$
\mathcal{F}_{s}(\vec{\xi} ; t)=l_{s}\left(1-\sum_{j} u_{s \rightarrow j}^{\text {out }}\right) \xi_{s}^{2}+\left[\sum_{j} l_{s} u_{s \rightarrow j}^{\text {out }} \xi_{j}^{\text {out }}-\left(l_{s}+D_{s}\right)\right] \xi_{s}+D_{s} .
$$

The dependence on time on the right-hand side comes from the fact that the kinetic rates such as birth and death rate can in general be functions of time. In order to evaluate the function $\Psi\left(\vec{\xi}^{\prime} ; t^{\prime}\right)$ at some point $\vec{\xi}^{\prime}=\left(\xi_{0}^{\prime}, \ldots, \xi_{n}^{\prime}\right)$, we "reverse the time" by the change of variables, $t \rightarrow t^{\prime}-t$, and solve the following initial value problem:

$$
\begin{aligned}
\dot{\xi}_{s} & =\mathcal{F}_{s}\left(\vec{\xi} ; t^{\prime}-t\right), \\
\xi_{s}(0) & =\xi_{s}^{\prime} .
\end{aligned}
$$

Note that the coefficients on the right-hand side can be time-dependent. The procedure of time-reversal in the particular case relevant for this paper is described in the next section. The probability generating function is obtained by

$$
\Psi\left(\vec{\xi}^{\prime} ; t^{\prime}\right)=\prod_{s=0}^{n}\left[\xi_{s}\left(t^{\prime}\right)\right]^{M_{s}},
$$

where on the right-hand side the functions $\xi_{s}(t)$ are solutions of system (3) and the constants $M_{s}$ are the initial abundances of mutants of type $s$. In the rest of this paper we will assume for simplicity that initially there are $M_{0}$ cells of type $s=00$ and zero cells of any other type.

In our particular case of four mutant classes, we have four equations:

$$
\begin{aligned}
\dot{\xi}_{00}= & l_{00}\left(1-u_{1}-u_{2}-u_{12}\right) \xi_{00}^{2}+\left(l_{00}\left(u_{1} \xi_{10}+u_{2} \xi_{01}+u_{12} \xi_{11}\right)\right. \\
& \left.-\left(l_{00}+D_{00}\right)\right) \xi_{00}+D_{00}, \\
\dot{\xi}_{10}= & l_{10}\left(1-u_{2}-u_{12}\right) \xi_{10}^{2}+\left(l_{10}\left(u_{2}+u_{12}\right) \xi_{11}-\left(l_{10}+D_{10}\right)\right) \xi_{10}+D_{10}, \\
\dot{\xi}_{01}= & l_{01}\left(1-u_{1}-u_{12}\right) \xi_{01}^{2}+\left(l_{01}\left(u_{1}+u_{12}\right) \xi_{11}-\left(l_{01}+D_{01}\right)\right) \xi_{01}+D_{01}, \\
\dot{\xi}_{11}= & l_{11} \xi_{11}^{2}-\left(l_{11}+D_{11}\right) \xi_{11}+D_{11},
\end{aligned}
$$

where the time-dependence of the coefficients is implicit.

\subsection{Modeling Treatment Strategies}

Different treatment strategies define the values of the death rates, $D_{s}$, at different moments of time:

$$
D_{s}=d_{s}+h_{s}(t) \text {, }
$$

where the coefficients $d_{s}$ are natural death rates of the cancer cells, and $h_{s}(t)$ are the drug-induced cell death rates. The functions $h_{s}(t)$ depend on the particular protocol used. As different drugs are applied, the "strength" of each drug, which depends on the concentration of the drug in the patient's blood, changes as some smooth function 
of time. The exact shape of these functions, and therefore, the shape of $h_{s}(t)$, depends not only on the treatment strategy (that is, whether drugs are applied in combination, or cyclically), but also on the way the drugs are administered, and on how quickly they are absorbed. For example, it can be assumed that $h_{s}(t)$ for a susceptible class reaches a maximum sometime after the drug is taken, and decays until the next administration of the drug. However, in this paper we simplify this picture by assuming that the functions $h_{s}(t)$ are piecewise-constant. They are assumed to have a constant nontrivial value for all the susceptible classes as long as the patient is treated with a given drug, and they become zero after the drug is discontinued. For the effects of pharmacokinetics on the dynamics of treatment, see Gaffney (2005).

We proceed to describe a cyclic two-drug treatment protocol, see Fig. 1(b). Let us suppose that treatment starts at time $t_{*}$. Then, for $0<t<t_{*}$, we have $D_{s}=d_{s}$; normally, $d_{s}<l_{s}$, that is, the cancer is assumed to grow stochastically before treatment. At time $t_{*}$, the first drug is applied for a length of time $\Delta t_{1}$. During this time, we have

$$
D_{00}=d_{00}+h_{1}, \quad D_{10}=d_{10}, \quad D_{01}=d_{01}+h_{1}, \quad D_{11}=d_{11},
$$

where $h_{1}$ is the drug-induced death rate for drug 1; see Fig. 1(a), on the left. After time $t_{*}+\Delta t_{1}$, drug 2 is applied for the duration $\Delta t_{2}$, resulting in

$$
D_{00}=d_{00}+h_{2}, \quad D_{10}=d_{10}+h_{2}, \quad D_{01}=d_{01}, \quad D_{11}=d_{11},
$$

for $t_{*}+\Delta t_{1}<t<t_{*}+\Delta t_{1}+\Delta t_{2}$ (Fig. 1(b), on the right). Here, $h_{2}$ denotes the drug-induced death rate of drug 2 . After that, treatment is again switched to drug 1 for duration $\Delta t_{1}$, and so on, for a total time duration $T_{\text {treat }}$, with a total of $2 \mathcal{N}$ cycles.

In this paper we assume that the division rates, $l_{s}$, and the death rates, $d_{s}$, of cells are time-independent. We will further assume that some of the coefficients in (6)-(9) are the same, namely, that $l_{s}=l$ and $d_{s}=d$.

\subsection{Probability of Treatment Success}

In our analysis we will be concerned with the probability of treatment success, which is assumed to be the same as the probability of extinction of the colony as a result of treatment.

To begin with, let us consider the quantity

$$
\varphi_{0, \ldots, 0}(t)=\Psi(\overrightarrow{0} ; t) .
$$

It has the meaning of having zero cells of all types at time $t$. To evaluate this function, we will use the general formula (5) with $M_{s}=0$ for $s \neq 00$ :

$$
\Psi(\overrightarrow{0} ; t)=\xi_{00}^{M_{0}}(t),
$$

where $\xi_{00}$ is the solution of system (6)-(9) with the initial conditions

$$
\xi_{00}=\xi_{10}=\xi_{01}=\xi_{11}=0 .
$$

The function $\varphi_{0, \ldots, 0}$ is related to the probability of treatment success. It includes the scenario where the colony goes extinct spontaneously (this happens with probability 
$\left.(d / l)^{M_{0}}\right)$, as well as the scenario where the tumor grows and is subsequently treated successfully. The latter process has the meaning of the probability of treatment success. In other words, we have

$$
\varphi_{0, \ldots, 0}=(d / l)^{M_{0}}+\left(1-(d / l)^{M_{0}}\right) P_{\text {success }}(t) .
$$

The quantity $P_{\text {success }}(t)$ is the focus of our study; it excludes the scenarios where the colony goes extinct spontaneously. We have

$$
P_{\text {success }}(t)=\frac{\xi_{00}^{M_{0}}(t)-(d / l)^{M_{0}}}{1-(d / l)^{M_{0}}} .
$$

The solution $\xi_{00}(t)$ is obtained from system (6)-(9). It consists of coupled nonlinear equations of the Riccati type, and will be solved numerically. This system is characterized by piecewise-constant coefficients, and as a consequence, the time-reversal procedure involved in the solution requires the following approach. Suppose for simplicity that the total duration of treatment equals $\mathcal{N}\left(\Delta t_{1}+\Delta t_{2}\right), \mathcal{N} \in I$; that is, it includes an integer and equal number of cycles of each drug. We start by solving system (6)-(9) with constant coefficients given by (11) and with initial conditions (12), and find the solution corresponding to the time $\Delta t_{2}$. Then we plug these values as initial conditions for system (6)-(9) with coefficients (10) and solve the equations for time duration $\Delta t_{1}$. Next, we use the resulting functions as the initial conditions and repeat the process for the total of $2 \mathcal{N}$ alternating cycles. This procedure corresponds to the ("reversed") time-variable changing from the end of treatment (physical time $\left.t_{*}+\mathcal{N}\left(\Delta t_{1}+\Delta t_{2}\right)\right)$ back to time $t_{*}$. Finally, we plug the obtained values in the same system with coefficients corresponding to the pre-treatment conditions $\left(D_{s}=d_{s}\right.$ for all $s$ ) and find the solution at $t=t_{*}$. The obtained function $\xi_{00}$ is used in formula (13).

The limiting value of the probability of treatment success,

$$
\lim _{t \rightarrow \infty} P_{\text {success }}(t)
$$

is of a particular interest. This approximation corresponds to long-term treatment strategies where the drugs are used long enough for all the susceptible types to be eliminated. The limiting procedure in our simulations corresponds to the number of cycles, $2 \mathcal{N}$, increasing to infinity. In practical terms, we perform the numerical procedure described above until the solution of the system corresponding to odd (even) cycles stops changing (within a prescribed precision).

The time of the start of treatment, $t_{*}$, can be related to the initial colony size, $N$, by the deterministic relationship,

$$
N=M_{0} e^{(l-d) t_{*}} .
$$

This is an approximation (Komarova et al. 2007), by which we replace a "fixed-size problem" (a problem where we know the tumor size at the start of treatment) with a much easier "fixed-time problem" (a problem where we know the time that elapsed from the beginning of tumor growth until the start of treatment). We will use formula (15) throughout the paper for simplicity. 
We will discuss two characteristics of drugs, their potency and their activity spectra. In terms of our modeling approach, drug potency is reflected in the drug-induced cell death rate characteristic of each drug, where higher potencies are correlated with higher values of $h_{s}$ for susceptible mutant classes. Drugs with broader activity spectra will be characterized by smaller mutation rates, $u$, with which mutants resistant to the drugs are generated. The more specific, or narrow, drugs correspond to larger mutation rates associated with the generation of resistant mutants.

\subsection{Finding the Optimal Strategy}

The optimal strategy is the pair $\left(\Delta t_{1}, \Delta t_{2}\right)$ which corresponds to the highest probability of treatment success for a given set of parameters. In order to find the optimal strategy for given parameters, we need to solve system (6)-(9) with initial conditions (12), and obtain the value $\xi_{00}(t)$, which is then used in formulas (13) or (14). Once this is done for a number of choices $\left(\Delta t_{1}, \Delta t_{2}\right)$, we have to find the pair of cycle durations that give the maximum probability of treatment success.

The numerical values of the probabilities of treatment success will depend on the parameters such as $l-d$ (the net growth of untreated cells), the mutation rates, and $N$ (the tumor size at the beginning of treatment). However, in the model presented here, the optimal strategy (that is, the optimal choice of $\Delta t_{1}$ and $\Delta t_{2}$ ) turns out to be independent on the tumor size, $N$.

The tumor size, $N$, enters the calculations of the probability of treatment success through formula (15), and defines the time $t_{*}$ of the pre-treatment stage of the solution. This stage is defined by the following initial value problem:

$$
\begin{aligned}
\dot{\xi}_{i} & =\mathcal{G}_{i}(\vec{\xi}), \quad 0 \leq i \leq n, 0<t \leq t_{*}, \\
\xi_{i}(0) & =y_{i},
\end{aligned}
$$

where the first equation is system (6)-(9) with $D_{i}=d$ and $l_{i}=l$, and the initial conditions $y_{i}$ are obtained by solving system (6)-(9) the appropriate number of times with the coefficients defined by the cyclic treatment stages. We need to find the probability of treatment success, which is a monotonically increasing function of $\xi_{00}$, as a function of $\Delta t_{1}$ and $\Delta t_{2}$. The information about $\Delta t_{1}$ and $\Delta t_{2}$ is contained only in the initial conditions, $\vec{y}=\left(y_{0}, \ldots, y_{n}\right)$. Therefore, we need to study the behavior of the quantity $\xi_{00}$ as a function of $\vec{y}$; we will denote this as $\xi_{00}^{\vec{y}}$. Let us pick two initial vectors, $\vec{y}_{1}$ and $\overrightarrow{y_{2}}$. We would like to show that if for some time $t_{1}, \xi_{00}^{\vec{y}_{1}}\left(t_{1}\right)>\xi_{00}^{\vec{y}_{2}}\left(t_{1}\right)$, then for any other time, $t_{2}$, we also have: $\xi_{00}^{\vec{y}_{1}}\left(t_{2}\right)>\xi_{00}^{\vec{y}_{2}}\left(t_{2}\right)$. In other words, the timeevolution prescribed by the system preserves the sign of the derivative of the solutions with respect to the initial conditions.

In order to show that this is indeed the case, let us denote $\lambda_{i k}=\partial \xi_{i} / \partial y_{k}$, and write down the initial value problems for these quantities:

$$
\begin{aligned}
\dot{\lambda}_{i k} & =\sum_{j} \frac{\partial \mathcal{G}_{i}}{\partial y_{j}} \lambda_{j k}, \\
\lambda_{i k}(0) & =\delta_{i k},
\end{aligned}
$$


where we simply differentiated (16)-(17) with respect to $y_{k}$, and used the notation $\delta_{i k}$ for the Kronecker's delta. Equations for $\lambda_{i k}$ comprise a homogeneous linear system with nonnegative initial conditions. It follows that the solutions of this system remain nonnegative. Therefore, if a given strategy $\left(\Delta t_{1}, \Delta t_{2}\right)$ corresponds to the maximum probability of treatment success for a given value $N$ (and the corresponding time $t_{*}$ before treatment), the same strategy is optimal for any tumor size $N$.

\subsection{The Limit of Very Short Cycles: The Average Combination Treatment}

Let us assume that $\Delta t_{2}=\alpha \Delta t_{1}$ with $\alpha>0$, and consider the limit where $\Delta t_{2} \rightarrow 0$. We can show that the cyclic treatment corresponds to the following continuous treatment:

$$
h_{00}=\frac{h_{1}+\alpha h_{2}}{1+\alpha}, \quad h_{10}=\frac{h_{2} \alpha}{1+\alpha}, \quad h_{01}=\frac{h_{1}}{1+\alpha}, \quad h_{11}=0 .
$$

This is equivalent to a simultaneous (combination) treatment with two drugs of individual strengths $\frac{h_{2} \alpha}{1+\alpha}$ and $\frac{h_{1}}{1+\alpha}$, and combined strength equal to the sum of the two individual strengths. We will refer to this treatment regime as the "average combination treatment." In the case where $\Delta t_{1}=\Delta t_{2}$, the values of the drug-induced death rates in the limiting combination treatment are $h_{00}=\left(h_{1}+h_{2}\right) / 2, h_{01}=h_{1} / 2, h_{10}=h_{2} / 2$, $h_{11}=0$; the corresponding diagram is presented in Fig. 1(c).

\section{Deterministic Modeling of Cyclic Treatments}

Here we create a simplified, deterministic framework for studying the effects of cyclic drug treatments. We start by writing down the equations for the expected numbers of different types of resistant mutants, which we call $x_{s}$ :

$$
\begin{aligned}
& \dot{x}_{00}=\left(l\left(1-u_{1}-u_{2}-u_{12}\right)-d-h_{00}\right) x_{00}, \\
& \dot{x}_{01}=\left(l\left(1-u_{1}-u_{12}\right)-d-h_{01}\right) x_{01}+l u_{2} x_{00}, \\
& \dot{x}_{10}=\left(l\left(1-u_{2}-u_{12}\right)-d-h_{10}\right) x_{10}+l u_{1} x_{00}, \\
& \dot{x}_{11}=(l-d) x_{11}+l\left[\left(u_{2}+u_{12}\right) x_{10}+\left(u_{1}+u_{12}\right) x_{01}+u_{12} x_{00}\right],
\end{aligned}
$$

where $l$ and $d$ are the division and death rates of the cells, and $h_{s}$ is the drug-induced death rate for resistance type $s$; the coefficients $h_{s}$ change depending on the treatment phase.

We are interested in the production of fully-resistant mutants, because (for longterm treatments) such mutants are the reason for treatment failure. These mutants are produced by mutations of partially-resistant mutants, and also, in the presence of cross-resistance, by mutations of fully-susceptible mutants. This happens both before the treatment starts and after it starts. The former process is treatmentindependent. Therefore, in order to evaluate the effectiveness of different treatment protocols, it is sufficient to only consider the latter process. The production 
of fully-resistant mutants is described by the second term on the right-hand side of (24). The total amount of doubly-resistant mutants produced after treatment starts is given by

$$
F=\int_{t_{*}}^{T_{\text {treat }}} l\left[\left(u_{2}+u_{12}\right) x_{10}+\left(u_{1}+u_{12}\right) x_{01}+u_{12} x_{00}\right] d t,
$$

where $t_{*}$ is the time-point where therapy is first applied, and $T_{\text {treat }}$ is the total treatment duration.

\subsection{Production of Fully-Resistant Mutants from Partially-Resistant Mutants}

Let us find the functions $x_{10}(t)$ and $x_{01}(t)$ by solving (22) and (23) for $t>t_{*}$. In our calculations, we will only consider the highest-order contributions in terms of the mutation rates.

Let us denote by $A\left(u_{1}\right)$ and $A\left(u_{2}\right)$ the expected number of mutants of types (10) and (01) at the start of treatment. These quantities can be calculated from equations similar to system (21)-(24), formulated for the pre-treatment period. In the limit of small mutation rates, we have

$$
A(u) \approx N \log N u,
$$

where $N=M_{0} e^{(l-d) t_{*}}$ is the number of cells at the beginning of treatment. Suppose that at the start of treatment, we have $A\left(u_{2}\right)$ mutants of type $(01)$ and $A\left(u_{1}\right)$ mutants of type (10). Starting at $t=t_{*}$, we treat with drug 1 for time duration $\Delta t_{1}$. This is followed immediately by treatment with drug 2 for time duration $\Delta t_{2}$, then again by treatment with drug 1 for time duration $\Delta t_{1}$, and so on, for an infinite number of cycles.

Let us denote $\gamma=l-d$. Then the average numbers of partially resistant mutants, $x_{10}(t)$ and $x_{10}(t)$, can be found as follows. We define quantities $x_{10}^{(i)}$ and $x_{01}^{(i)}$ for $i=1,2, \ldots$ by means of the differential equations:

$$
\begin{aligned}
& \dot{x}_{01}^{(0)}=\left(\gamma-h_{1}\right) x_{01}, \quad t_{*}<t<t_{*}+\Delta t_{1}, \quad x_{01}\left(t_{*}\right)=A\left(u_{2}\right) ; \\
& \dot{x}_{10}^{(0)}=\gamma x_{10}, \quad t_{*}<t<t_{*}+\Delta t_{1}, \quad x_{10}\left(t_{*}\right)=A\left(u_{1}\right) ; \\
& \dot{x}_{01}^{(1)}=\gamma x_{01}, \quad t_{*}+\Delta t_{1}<t<t_{*}+\Delta t_{1}+\Delta t_{2}, \quad x_{01}\left(t_{*}\right)=x_{01}^{(0)}\left(t_{*}+\Delta t_{1}\right) ; \\
& \dot{x}_{10}^{(1)}=\left(\gamma-h_{2}\right) x_{10}, \quad t_{*}+\Delta t_{1}<t<t_{*}+\Delta t_{1}+\Delta t_{2}, \quad x_{10}\left(t_{*}\right)=x_{10}^{(0)}\left(t_{*}+\Delta t_{1}\right) ;
\end{aligned}
$$

To write down the equations for the general cycle $i$, it is convenient to define the intervals

$$
U_{i}= \begin{cases}{\left[t_{*}+\frac{i}{2}\left(\Delta t_{1}+\Delta t_{2}\right), t_{*}+\frac{i}{2}\left(\Delta t_{1}+\Delta t_{2}\right)+\Delta t_{1}\right),} & i \text { is even } \\ {\left[t_{*}+\frac{i-1}{2}\left(\Delta t_{1}+\Delta t_{2}\right)+\Delta t_{1}, t_{*}+\frac{i+1}{2}\left(\Delta t_{1}+\Delta t_{2}\right)\right),} & i \text { is odd }\end{cases}
$$


Each interval equals a single cycle of a drug; even values of $i$ correspond to cycles of drug 1 and odd values of $i$-to cycles of drug 2 (more precisely, interval $U_{i}$ corresponds to the $(i / 2+1)$ th application of drug 1 for even values of $i$, and to the $((i-1) / 2+1)$ th application of drug 2 if $i$ is odd $)$. The dynamics of cells types are described by an initial value problem within each interval, $U_{i}$. We have, if $i$ is even:

$$
\begin{aligned}
\dot{x}_{01}^{(i)}=\left(\gamma-h_{1}\right) x_{01}, \quad \dot{x}_{10}^{(i)} & =\gamma x_{10}, \quad t \in U_{i}, \\
x_{01}\left(t_{*}+\frac{i}{2}\left(\Delta t_{1}+\Delta t_{2}\right)\right) & =x_{01}^{(i-1)}\left(t_{*}+\frac{i}{2}\left(\Delta t_{1}+\Delta t_{2}\right)\right), \\
x_{10}\left(t_{*}+\frac{i}{2}\left(\Delta t_{1}+\Delta t_{2}\right)\right) & =x_{10}^{(i-1)}\left(t_{*}+\frac{i}{2}\left(\Delta t_{1}+\Delta t_{2}\right)\right) ;
\end{aligned}
$$

and if $i$ is odd:

$$
\begin{aligned}
& \dot{x}_{01}^{(i)}=(\gamma) x_{01}, \quad \dot{x}_{10}^{(i)}=\left(\gamma-h_{2}\right) x_{10}, \quad t \in U_{i}, \\
& x_{01}\left(t_{*}+\frac{i-1}{2}\left(\Delta t_{1}+\Delta t_{2}\right)+\Delta t_{1}\right)=x_{01}^{(i-1)}\left(t_{*}+\frac{i-1}{2}\left(\Delta t_{1}+\Delta t_{2}\right)+\Delta t_{1}\right), \\
& x_{10}\left(t_{*}+\frac{i-1}{2}\left(\Delta t_{1}+\Delta t_{2}\right)+\Delta t_{1}\right)=x_{10}^{(i-1)}\left(t_{*}+\frac{i-1}{2}\left(\Delta t_{1}+\Delta t_{2}\right)+\Delta t_{1}\right) .
\end{aligned}
$$

For any given value of $t>t_{*}$, we can find $i$ such that $t \in U_{i}$. Then $x_{10}(t)=x_{10}^{(i)}(t)$ and $x_{01}(t)=x_{01}^{(i)}(t)$. The quantity of interest is the total amount of the fully resistant types produced, which, after $2 \mathcal{N}$ cycles, is given by

$$
F_{1 \rightarrow 2}(\mathcal{N})=l \sum_{i=0}^{2 \mathcal{N}} \int_{t \in U_{i}}\left[\left(u_{1}+u_{12}\right) x_{01}^{(i)}(t)+\left(u_{2}+u_{12}\right) x_{10}^{(i)}(t)\right] d t
$$

where the subscript $1 \rightarrow 2$ indicates that the mechanism of resistance generation considered here is a mutation of cells resistant to only one drug, into cells resistant to two drugs. Solving (25) and (26) and integrating, we obtain:

$$
\begin{aligned}
& F_{1 \rightarrow 2}(\mathcal{N}) / l \\
& =\frac{A\left(u_{2}\right)\left(u_{1}+u_{12}\right)\left(1-e^{\mathcal{N}\left[\left(\gamma-h_{1} 1\right) \Delta t_{1}+\gamma \Delta t_{2}\right]}\right)}{\gamma}\left(\frac{\left(e^{\gamma \Delta t_{1}}-e^{h_{1} \Delta t_{1}}\right) h_{1}}{\left(e^{h_{1} \Delta t_{1}}-e^{\gamma\left(\Delta t_{1}+\Delta t_{2}\right)}\right)\left(\gamma-h_{1}\right)}-1\right) \\
& \quad+\frac{A\left(u_{1}\right)\left(u_{2}+u_{12}\right)\left(1-e^{\mathcal{N}\left[\gamma \Delta t_{1}+\left(\gamma-h_{2}\right) \Delta t_{2}\right]}\right)}{h_{2}-\gamma}\left(\frac{e^{h_{2} \Delta t_{2}} h_{2}\left(e^{\gamma \Delta t_{1}}-1\right)}{\gamma\left(e^{h_{2} \Delta t_{2}}-e^{\gamma\left(\Delta t_{1}+\Delta t_{2}\right)}\right)}+1\right) .
\end{aligned}
$$

Let us consider the limit of long treatments, $\mathcal{N} \rightarrow \infty$. For convergence, we need to assume that $\left(\gamma-h_{1}\right) \Delta t_{1}+\gamma \Delta t_{2}<0$ and $\gamma \Delta t_{1}+\left(\gamma-h_{2}\right) \Delta t_{2}<0$ (see Sect. 3.4 for 
an explanation of these assumptions). Then we have:

$$
\begin{aligned}
F_{1 \rightarrow 2} / l= & \frac{A\left(u_{2}\right)\left(u_{1}+u_{12}\right)}{\gamma}\left(\frac{\left(e^{\gamma \Delta t_{1}}-e^{h_{1} \Delta t_{1}}\right) h_{1}}{\left(e^{h_{1} \Delta t_{1}}-e^{\gamma\left(\Delta t_{1}+\Delta t_{2}\right)}\right)\left(\gamma-h_{1}\right)}-1\right) \\
& +\frac{A\left(u_{1}\right)\left(u_{2}+u_{12}\right)}{h_{2}-\gamma}\left(\frac{e^{h_{2} \Delta t_{2}} h_{2}\left(e^{\gamma \Delta t_{1}}-1\right)}{\gamma\left(e^{h_{2} \Delta t_{2}}-e^{\gamma\left(\Delta t_{1}+\Delta t_{2}\right)}\right)}+1\right) .
\end{aligned}
$$

\subsection{Production of Fully-Resistant Mutants from Fully-Susceptible Cells}

Let us next consider the production of cells of type $x_{11}$ directly from the cells of type $x_{00}$ by means of mutations with the rate $u_{12}$. The equations for $x_{00}$ are $\dot{x}_{00}=$ $\left(\gamma-h_{1}\right) x_{00}$ and $\dot{x}_{00}=\left(\gamma-h_{2}\right) x_{00}$ for odd and even cycles, respectively. Solving these equations and integrating, we can calculate the additional contribution to the function $F$ coming from cross-resistance. The result in the limit of long treatments is:

$$
\begin{aligned}
F_{0 \rightarrow 2} / l & =u_{12} \int_{0}^{\infty} x_{00}(t) d t \\
& =u_{12} N \frac{e^{h_{1} \Delta t_{1}+h_{2} \Delta t_{2}}\left(\gamma-h_{2}\right)-e^{\gamma\left(\Delta t-1+\Delta t_{2}\right)}\left(\gamma-h_{1}\right)+e^{\gamma \Delta t_{1}+h_{2} \Delta t_{2}}\left(h_{2}-h_{1}\right)}{\left(e^{\gamma\left(\Delta t_{1}+\Delta t_{2}\right)}-e^{h_{1} \Delta t_{1}+h_{2} \Delta t_{2}}\right)\left(\gamma-h_{1}\right)\left(\gamma-h_{2}\right)},
\end{aligned}
$$

where $N$ is the number of cells at the beginning of treatment; as before, we assume that the mutation rates are small.

\subsection{Optimization of Treatment Strategies}

The deterministic method of finding the optimal treatment strategy is based on the minimization of the objective function,

$$
F=F_{1 \rightarrow 2}+F_{0 \rightarrow 2}
$$

with respect to parameters $\Delta t_{1}$ and $\Delta t_{2}$. In the absence of cross-resistance, fully resistant mutants are produced only by partially-resistant mutants, and we have $F=F_{1 \rightarrow 2}$ with $u_{12}=0$. In the presence of cross-resistance, both mechanisms of fully-resistant mutant production are in place.

The objective function $F$ is correlated with the probability of treatment failure. Its minimum, as a function of $\Delta t_{1}$ and $\Delta t_{2}$, optimizes the treatment protocol. Finding this minimum is a much easier task compared to that of the stochastic method, where we need to maximize the probability of treatment success, which can only be calculated numerically (e.g. by means of the Runge-Kutta method of solving ordinary differential equations for the characteristics). Some interesting properties of the objective function are discussed in Appendix A. 


\subsection{Method Applicability and Mutually Strong Drugs}

The deterministic method described here is very intuitive and easy to implement compared to the stochastic method. While it does not work well for short treatments (see Sect. 6), in the limit of long treatments it describes reality remarkably well.

To find the conditions of applicability of the deterministic method, let us solve linear differential equations (25) and (26) to find the dynamics of partially-resistant mutants under treatment conditions. After $2 \mathcal{N}$ cycles, that is, for $t_{\mathcal{N}}=\mathcal{N}\left(\Delta t_{1}+\Delta t_{2}\right)$, we have

$$
x_{01}\left(t_{\mathcal{N}}\right)=A\left(u_{2}\right) e^{\mathcal{N}\left[\left(\gamma-h_{1}\right) \Delta t_{1}+\gamma \Delta t_{2}\right]}, \quad x_{10}\left(t_{\mathcal{N}}\right)=A\left(u_{1}\right) e^{\mathcal{N}\left[\gamma \Delta t_{1}+\left(\gamma-h_{2}\right) \Delta t_{2}\right]} .
$$

Therefore, in order for the treatment in the absence of doubly-resistant mutants to work, we need to require that

$$
h_{1}, h_{2}>\gamma \quad \text { and } \quad \frac{\gamma}{h_{2}-\gamma}<\frac{\Delta t_{2}}{\Delta t_{1}}<\frac{h_{1}-\gamma}{\gamma} .
$$

If conditions (32) are satisfied, functions $x_{10}(t)$ and $x_{01}(t)$ will on average decay. Conditions (32) are equivalent to condition

$$
\frac{1}{h_{1}}+\frac{1}{h_{2}}<\frac{1}{\gamma},
$$

which can be viewed as a requirement for the two drugs to be sufficiently strong (compared to the colony growth-rate $\gamma$ ) such that they can eliminate a population of partially resistant mutants. We will refer to drugs that satisfy condition (33) as mutually strong drugs.

We conclude that the deterministic method as described by (28)-(30) is applicable if the drugs have a sufficient potency to satisfy condition (32). By construction, the present framework handles only the cases where treatment failure occurs because of the production of doubly-resistant mutants. In the following sections we will further demonstrate the power of the deterministic method, and also show how it can fail.

\section{Optimal Treatment Strategies for Different Choices of Drugs}

We start the analysis by a numerical exploration of treatment strategies for cyclic treatments with two drugs of different strengths. For each pair of drug-induced death rates, $\left(h_{1}, h_{2}\right)$, we use the stochastic method of Sect. 2 to evaluate the probability of treatment success for different values of $\Delta t_{1}$ and $\Delta t_{2}$, in the limit of long treatments. We then find the optimal strategy for each pair $\left(h_{1}, h_{2}\right)$, and calculate the corresponding maximum probability of treatment success. The results are presented in Fig. 2, which shows schematically several regions on the $h_{1}-h_{2}$ diagram whose relatively sharp boundaries are defined by the difference in the treatment success probability for the optimal strategy. In what follows, we will use the notation

$$
\alpha=\frac{\Delta t_{2}}{\Delta t_{1}} .
$$


Fig. 2 Optimal cyclic treatments for two drugs with different drug potencies and equal activity spectra. The two axes are the strengths of the drugs, $h_{1}$ and $h_{2}$. The lines correspond to $h_{1}=\gamma, h_{2}=\gamma$, $h_{1}=h_{2}$ and $h_{1}^{-1}+h_{2}^{-1}=\gamma^{-1}$. The regions in the diagram marked by A, B, C, D, E, and F are characterized by different optimal treatment strategies, and different maximal probabilities of treatment success. See text for more details. The parameters are $l=1, d=0, h_{12}=0$, $u=10^{-6}, N=10^{10}$, $T_{\text {treat }} \rightarrow \infty$

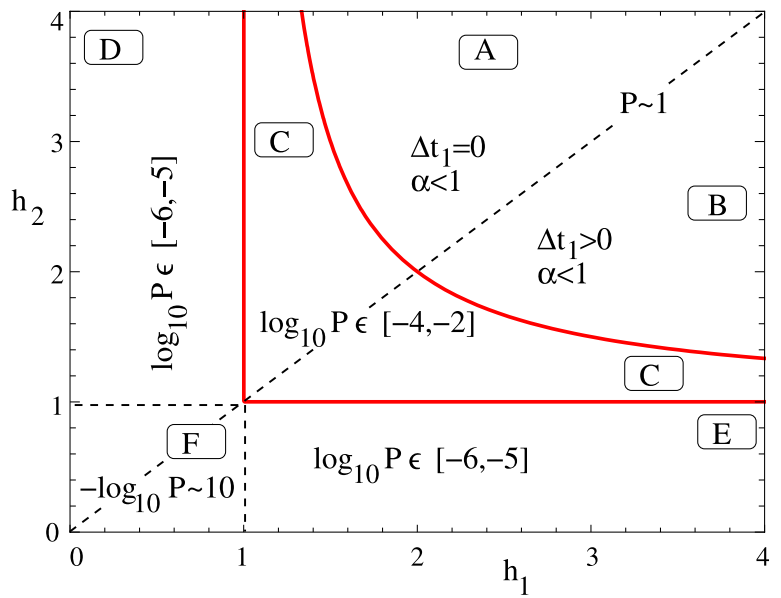

Regions A and B are characterized by the highest probabilities of treatment success, which for the particular choice of parameters in Fig. 2 are of the order one. There, we have $h_{1}^{-1}+h_{2}^{-1}<\gamma^{-1}$, that is, the two drugs are mutually strong, see inequality (33). This condition means that one can design a cyclic treatment such that both types of partially-resistant mutants will be driven extinct, and the only reason for treatment failure is the production of fully-resistant, double-hit mutants. Parameters that correspond to the highest probability of treatment success in regions A and B satisfy

$$
\frac{\gamma}{h_{2}-\gamma}<\alpha<\frac{h_{1}-\gamma}{\gamma},
$$

see condition (32). Outside this region, the probability of treatment success drops by orders of magnitude.

For mutually strong drugs, region A corresponds to $h_{2}>h_{1}$ (Worst Drug First, WDF) and region $\mathrm{B}$ to $h_{2}<h_{1}$ (Best Drug First, BDF). In the following section we will show that in region $\mathrm{A}$, the optimal treatment strategy corresponds to $\Delta t_{1}=0$, and $\alpha<1$ (treat with the worst drug longer); in region $\mathrm{B}$ we have an optimal, nonzero $\Delta t_{1}$, and $\alpha>1$ (again, treat with the worst drug longer). Regions $\mathrm{A}$ and $\mathrm{B}$ are the only ones where condition (33) holds, and we can apply the deterministic method; most of the rest of the paper is devoted to studying this regime. In regions $\mathrm{C}, \mathrm{D}, \mathrm{E}$, and $\mathrm{F}$ the deterministic method predicts treatment failure. Consistently with that, the stochastic method demonstrates that the probability of treatment success for regions outside $\mathrm{A}$ and $\mathrm{B}$ is significantly lower than that for regions A and B. A qualitative description of numerical findings for regions outside $\mathrm{A}$ and $\mathrm{B}$ is presented below; some analysis of the system's behavior outside regions A and B is given in Appendix B.

Let us consider region $\mathrm{C}$ in Fig. 2, where $h_{1}, h_{2}>\gamma$, but $h_{1}^{-1}+h_{2}^{-1}<\gamma^{-1}$. In this region, each drug is strong enough to eliminate the mutants susceptible to it, if we apply the drug continuously (this is because each drug-induced death rate is larger than $\gamma$ ). However, in a cyclic treatment, we cannot reach extinction of both partially-resistant types, because condition (33) is violated. The maximum probability of treatment success in region $\mathrm{C}$ is several orders of magnitude smaller than that 
in regions A and B; for the particular parameter values of Fig. 2 it is between $10^{-4}$ and $10^{-2}$. In this regime, the probability of treatment success strongly depends on $h_{1}$, the strength of the first drug, and is much less sensitive to $h_{2}$. We observe that treatment strategies starting from the stronger of the drugs are always characterized by a higher probability of treatment success. When we start with the stronger of the drugs, the optimal strategy is to have only two cycles: during the fist interval $\Delta t_{1}$, we try to drive the number of type- $x_{01}$ mutants to low numbers (to increase the probability of their stochastic extinction); during the subsequent interval $\alpha \Delta t_{1}$, we eliminate the other mutant type. This strategy is quite different from the optimal strategies in regions $\mathrm{A}$ and $\mathrm{B}$, and typically the outcome is a much poorer success probability.

In regions $\mathrm{D}$ and $\mathrm{E}$, the probability of treatment success again drops by a couple of orders of magnitude (it is between $10^{-6}$ and $10^{-5}$ in Fig. 2). These regions are characterized by very weak drugs. Only the second drug in region D is capable of eliminating mutants susceptible to it (because there, $h_{1}<\gamma, h_{2}>\gamma$ ). Similarly, in region $\mathrm{E}$, only the first drug is strong enough to eliminate the susceptible cells. The best strategy in region $\mathrm{D}$ is therefore to keep the application of the first (weaker) drug as short as possible $\left(\Delta t_{1}=0\right)$, and treat with the second (stronger) drug for longer $(\alpha \rightarrow \infty)$. In region $\mathrm{E}$, the class of the most successful strategies includes those where we treat with the first (stronger) drug for a long time (large $\Delta t_{1}$ ), such that the mutants susceptible to this drug are eliminated. In this case, the duration of the treatment with the second (weak) drug is unimportant (the value of $\alpha$ does not affect the probability of treatment success). All the strategies described for regions $\mathrm{C}$, $\mathrm{D}$ and $\mathrm{E}$ are essentially one-drug treatment strategies where we only use the stronger of the drugs; the only way in which treatment can be successful for such strategies is if there are no mutants resistant to the stronger drug. In region $\mathrm{E}$, if the strength of the second drug is not too low, there is another class of strategies where the second drug is utilized at an optimal duration. A rigorous analysis of such strategies is beyond the scope of this paper.

Finally, in region $\mathrm{F}$ we have $h_{1}, h_{2}<\gamma$, that is, neither of the drugs is capable of eliminating susceptible mutants. In this region, the probability of treatment success is practically zero (and equals to the probability of tumor's spontaneous extinction). The only way to achieve better treatment success is to combine the two drugs (Komarova and Wodarz 2005).

We can see that for all practical purposes, the drugs can be considered effective in the context of a cyclic treatment if they satisfy condition (33). Moreover, condition (32) should be satisfied to achieve a reasonable treatment schedule. The rest of this paper's analysis is therefore devoted to regions A and B.

\section{Analysis of Drug Treatments with Mutually Strong Drugs}

\subsection{Drugs of Different Potencies}

Here we examine the case where the two mutually strong drugs have equal activity spectra, and differ by their potency. Therefore, in this section we will assume that condition (33) holds; we will further set $u_{1}=u_{2}$, and study the production of fully 
resistant mutants depending on the values $h_{1}$ and $h_{2}$. We will address the following questions:

1. In a cyclic treatment, which of the two drugs should be applied first?

2. What are the optimal durations of the cycles, $\Delta t_{1}$ and $\Delta t_{2}$ ?

We will first consider systems without cross-resistance by setting $u_{12}=0$, and then study the effects of cross-resistance.

To gain some understanding of the two-drug system, we first apply the deterministic approach, in the absence of cross-resistance, under the assumption of long treatment protocols. We would like to find the global minimum of the objective function, $F_{1 \rightarrow 2}$, given by (28) with $u_{1}=u_{2}=u$ and $u_{12}=0$, as a function $\Delta t_{1}$ and $\Delta t_{2}$. It turns out that the objective function $F$ is discontinuous as $\Delta t_{1} \rightarrow 0$ and $\Delta t_{2} \rightarrow 0$. To see this, let us take

$$
\Delta t_{2}=\alpha \Delta t_{1},
$$

where $\frac{\gamma}{h_{2}-\gamma}<\alpha<\frac{h_{1}-\gamma}{\gamma}$, see condition (32). The limiting value,

$$
\lim _{\Delta t_{1} \rightarrow 0} F /(l A(u) u)=\left(\frac{h_{1}}{(1+\alpha) \gamma}-1\right)^{-1}+\left(\frac{\alpha h_{2}}{(1+\alpha) \gamma}-1\right)^{-1},
$$

depends on $\alpha$. This is because the "average" treatment corresponding to drug strengths given by (20), depends on $\alpha$. Therefore, to visualize $F$, we will plot it as a function of $\Delta t_{1}$ and $\alpha$.

Figure 3(a) presents contour plots of the objective function $F$ (scaled) with respect to the length of the first cycle, $\Delta t_{1}$, and the ratio of the two cycle lengths, $\alpha$. The dashed contours represent the worst drug first (WDF) strategy, and the solid contours - the best drug first (BDF) strategy. The dots indicate the location of the minima of the function $F$. We observe that for the WDF strategies, the absolute minimum is reached at $\Delta t_{1}=0$ and $\alpha<1$, and corresponds to the average combination treatment. On the other hand, for the BDF strategies, the absolute minimum is achieved for a positive value of $\Delta t_{1}$ and $\alpha>1$. The value of the latter minimum is lower than the former one. Therefore, the optimal strategy, as predicted by the deterministic approximation, is to start treatment with the better drug, but to arrange for the worse drug to have a longer cycle.

The results presented above were obtained by using the deterministic method of Sect. 3. For more details of the analysis of the objective function, see Appendix A, and also Katouli (2009). In Appendix C we show that the predictions obtained by the deterministic method are in good agreement with the stochastic results.

Next, we include cross-resistance by setting $u_{12}>0$, and explore how this affects results such as those presented in Fig. 3(a). Examining the term $F_{0 \rightarrow 2}$ (29), we can see the following patterns:

- If $h_{1}>h_{2}$, then $F_{0 \rightarrow 2}$ is minimized by increasing $\Delta t_{1}$ and setting $\alpha=0$; for sufficiently large $\Delta t_{1}$, the dependence of $\alpha$ becomes weak.

- If $h_{1}<h_{2}$, then $F_{0 \rightarrow 2}$ is minimized by setting $\Delta t_{1}=0$ and increasing $\alpha$.

- The lowest minimum corresponds to the $h_{1}>h_{2}$ case. 
(a)

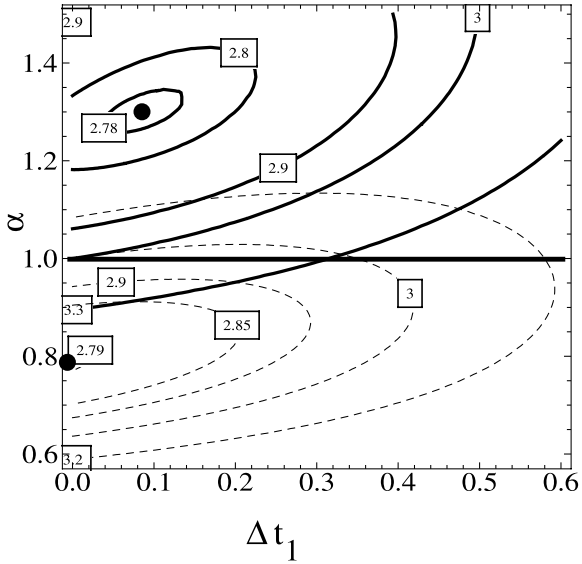

(b)

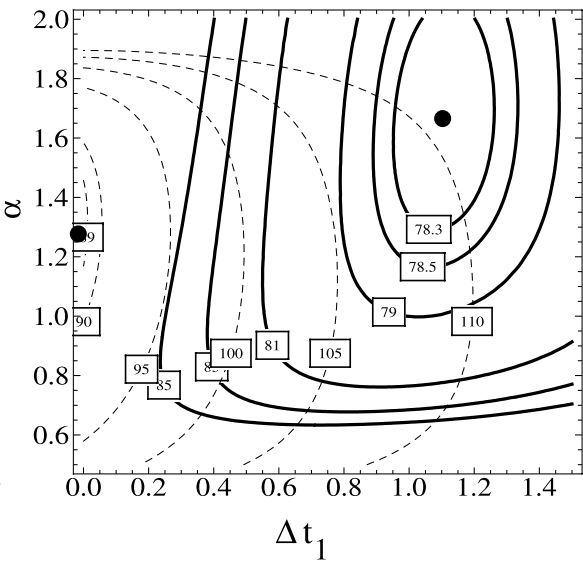

Fig. 3 Different drug potencies, equal activity spectra. Using the deterministic approximation, we present contour plots of the objective function $F /\left(l N \log N u_{1} u_{2}\right)$, (29), for the alternating strategies with the WDF (dashed lines), and the BDF (solid lines). The black dots indicate the minima (corresponding to the best $\left(\Delta t_{1}, \alpha\right)$ pair). (a) Without cross-resistance; (b) in the presence of cross-resistance. The parameters are $l=1, d=0, u_{12}=5000 u_{1} u_{2}$, and the drug-induced death rates are $3 \gamma$ and $4 \gamma$

Clearly, if we want to decrease the production of fully-resistant cells by means of mutations of $x_{00}$, we need to treat the fully-susceptible population with the stronger of the drug. If the constraint of cyclic treatments is imposed, then the best option is to start with the better of the drugs and keep the cycle duration of the better drug as high as possible; for sufficiently long $\Delta t_{1}$, the duration of the weaker drug cycle does not matter anymore, because most of the susceptible population will be extinct. However, if we start from the worse of the drugs, then it is optimal to keep the first cycle as short as possible, and the cycle corresponding to the better drug as long as possible.

These trends reinforce the result of Fig. 3(a) that the best strategy must start with the better drug, see Fig. 3(b). Further, the optimal cycle duration $\Delta t_{1}$ is expected to increase with respect to that of Fig. 3(a), solid lines, while the optimal $\alpha$ will not decrease (see Fig. 3(b)). On the other hand, if we start with the worse of the drugs (Fig. 3(a,b), dashed lines), then, if the effect of the cross-mutations is sufficiently strong, we expect the optimal $\alpha$ to become greater than 1 , that is, the duration of the better drug cycle should be longer.

All these results were obtained by using the deterministic approximation, and they have been confirmed by numerical simulations of the stochastic model (not shown). We obtained a good agreement between the two methods regarding the predicted optimal parameters $\Delta t_{1}$ and $\alpha$. A further analysis of the stochastic results shows that the difference between the best BDF protocol and the best WDF protocol in the limit of long treatment times is not large (usually, within 1\%). However it would be misleading to think that there is no real difference between BDF and WDF strategies. Firstly, in order to achieve an optimal treatment timing, the treatment parameters $\Delta t_{1}$ and $\alpha$ have to be optimized, and the choice of these parameter values strongly depends on whether we use a BDF or a WDF protocol. Secondly, the advantage of 
BDF protocols is not only that they yield a higher treatment success probability. They are also characterized by a finite (nonzero) optimal cycle duration, $\Delta t_{1}$. On the other hand as we have shown, WDF protocols have a zero optimal cycle duration, which is very difficult to implement in reality. Finally, as we will show in Sect. 6, the advantage in the probability of treatment success for BDF protocols increases significantly as the treatment durations become shorter.

\subsection{Drugs of Equal Potencies and Different Activity Spectra}

In this section we examine the case where the two mutually strong drugs have equal potencies $\left(h_{1}=h_{2}\right)$ but differ by their activity spectra, which corresponds to nonequal mutation rates, $u_{1} \neq u_{2}$. The drugs with broader (narrower) activity spectra correspond to the lower (higher) values of the mutation rate. We first consider the scenario without cross-resistance and then add the effect of cross-resistance.

Consider the deterministic method developed in Sect. 3, by setting $h_{1}=h_{2}$ and using different values for the mutation rates. In (28) which represents the average production of doubly-resistant mutants, the mutation rates enter in combinations $A\left(u_{1}\right) u_{2}$ and $A\left(u_{2}\right) u_{1}$, where $A(u)$ is the expected number of mutants generated at rate $u$ by the time the untreated colony grows to size $N$. The quantity $A\left(u_{1}\right)$ is obtained by solving the equations,

$$
\begin{aligned}
\dot{x}_{00} & =\left(l\left(1-u_{1}\right)-d\right) x_{00}, \quad \dot{x}_{10}=\left(l\left(1-u_{2}\right)-d\right) x_{10}+l u_{1} x_{00}, \\
x_{00}(0) & =M_{0}, \quad x_{10}(0)=0,
\end{aligned}
$$

such that $A\left(u_{1}\right)=x_{10}\left(t_{*}\right)$, and $M_{0} e^{(l-d) t_{*}}=N$. A similar algorithm applies for $A\left(u_{2}\right)$. Expanding the solution into the Taylor series in terms of the small mutation rates, we obtain,

$$
A\left(u_{1}\right)=u_{1} N \log N, \quad A\left(u_{2}\right)=u_{2} N \log N,
$$

and therefore $A\left(u_{1}\right) u_{2}=A\left(u_{2}\right) u_{1}$. This means that the objective function $F$ in (28) is symmetric with respect to the interchange of $u_{1}$ and $u_{2}$, and thus it follows that according to the deterministic method, the BDF and WDF strategies are equivalent. In other words, it does not matter which drug we start with. A contour plot of the objective function for a particular parameter set is presented in Fig. 4(a). The explanation is as follows. The mutants resistant to the drug with a higher mutation rate are created faster, so they are more abundant, but during the treatment they produce fully-resistant mutants with a lower intensity. The mutants resistant to the drug with a lower mutation rate are less abundant at the start, but they generate fully-resistant mutants faster. In the deterministic system, the two effects balance each other out, leading to the observed symmetry.

Our numerical results based on the stochastic model of Sect. 2 with $u_{12}=0$ are illustrated in Fig. 4(b). Shown are contour plots of the probability of treatment success, when the treatment starts with the drug with the higher mutation rate (solid lines) and the lower mutation rate (dashed lines). We observe that the two sets of contours are not identical in the stochastic method. In fact, if $u_{1}>u_{2}$, then the optimal treatment regime corresponds to $\Delta t=0$, and if $u_{1}<u_{2}$, then there is an optimal cycle duration 
(a)

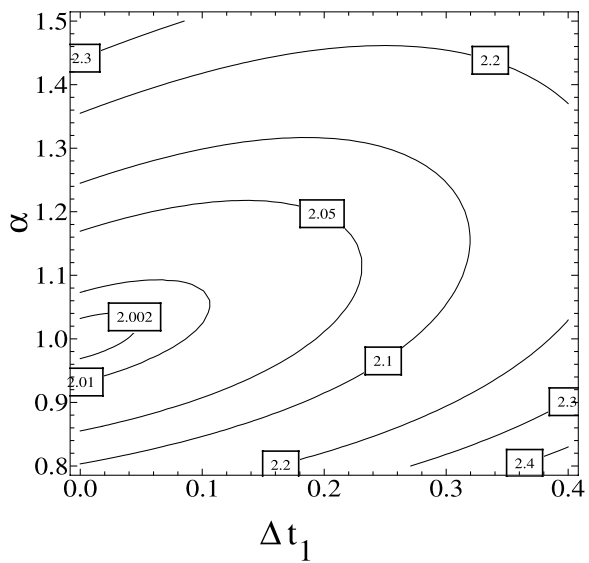

(b)

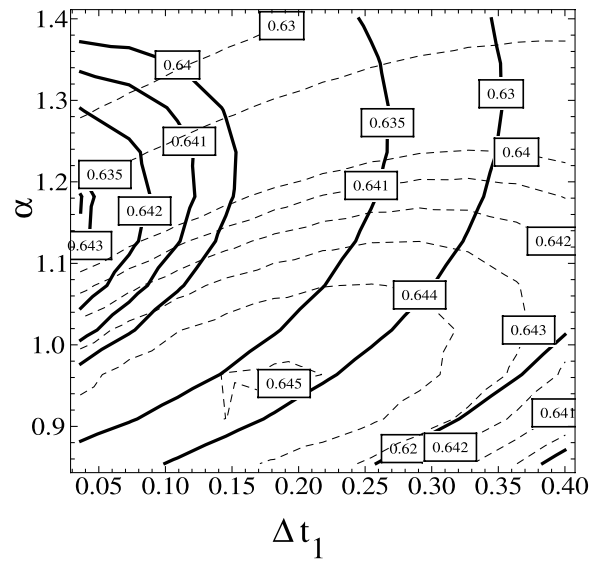

Fig. 4 Equal drug potencies, different activity spectra. (a) The contour plots of the objective function for the deterministic method. (b) Contour plots of the probability of treatment success (using the stochastic method) for the alternating strategies with the drug with a narrower activity spectrum first (solid contours), and the broader drug first (dashed contours). The mutation rates are $10^{-5}$ and $10^{-8}$, and the rest of the parameters are $l=1, d=0, h_{1,2}=4, N=10^{11}$

$\Delta t_{*}>0$. The probability of treatment success is higher in the case where $u_{1}<u_{2}$. That is, it is better to start with the drug with a broader activity spectrum.

The general conclusion is that for drugs of equal potency, the optimal treatment starts with the drug for which resistance is generated slower. In other words, it is best to start with the drug which is effective against the more abundant resistant mutants. The optimal cycle duration is nonzero, and the cycle length of the drug with the narrower activity spectrum is smaller than that of the drug with the broader activity spectrum.

Note that the difference between the two maxima in Fig. 4(b) is very small (in the third digit); this is a general trend for drugs of equal potency. Therefore, the deterministic result stating that the order of drugs is not important is correct to a certain approximation. However, the calculation of the optimal strategy (that is, the best values for $\Delta t_{1}$ and $\Delta t_{2}$ ) cannot be made by using the deterministic method. For an explanation of the slight asymmetry leading to the advantage of using broaderactivity drugs first, see Appendix D.

Next, we explore how cross-resistance influences the above results. For equal drug potencies, we apply the deterministic method of Sect. 3 in the context of crossresistance. The term $F_{0 \rightarrow 2}$, (29), under the assumption $h_{2}=h_{1}$, equals $F_{0 \rightarrow 2}=$ $N u_{12} /\left[l\left(h_{1}-\gamma\right)\right]$, that is, it does not depend on the cycle durations of the two drugs. This makes sense, because the production of fully-resistant mutants directly out of susceptible cells is unaffected by the mutation rates $u_{1}$ and $u_{2}$, and therefore, it does not matter which drug is used first, and what the cycle durations are. Computer simulations of the stochastic model with cross-resistance confirm that the influence of cross-resistance in the case of equal potency, different activity drugs is negligible (not shown). We predict that the optimal strategy is to start with a broader drug, and use it for longer periods of time. The only difference which cross-resistance makes 
is the weakening of the effect, that is, the flattening of the maximum of the treatment success probability. For high values of $u_{12}$, the primary reason for treatment failure becomes the creation of double mutants by cross-resistance, and therefore the exact treatment strategy (the timing and duration of the drugs) becomes less important, as it only affects the (secondary) process of double-mutant creation by two independent mutations.

\section{Short Treatment Times}

So far, we have been focusing on studying the limit of long treatments. This means that in the deterministic model, we considered the limit of an infinite number of cycles, $\mathcal{N} \rightarrow \infty$ in expressions such as (27), which yields the objective functions in (28), (29). In the stochastic simulations, we performed cycles of numerical integration of ODEs until the result did not change any longer. In this section we will address the situation where the treatment time is suboptimal. In other words, an increase in treatment time would lead to a sizable improvement in the chance of treatment success.

Let us fix a certain treatment time, $T_{\text {treat }}$, and vary the number of cycles, $2 \mathcal{N}$, used in the protocol. This is similar to varying the cycle duration in the case of "infinite" treatments. First we consider drugs of different potencies. We would like to know (i) what drug should be used first, (ii) what number of cycles, $2 \mathcal{N}$, should be implemented within the allocated treatment time, and (iii) what is the optimal cycle ratio for the two drugs. In Fig. 5 we considered two drugs: drug $A$ has potency $h=5 \gamma$ and drug $B$ has potency $h=3 \gamma$; the two drugs have the same activity spectra. The contour plots show the levels of the probability of treatment success calculated by using the stochastic methodology. Solid contours correspond to BDF strategies, and dashed contours-to WDF strategies. In Fig. 5(a), the treatment time is taken essentially infinite; in other words, doubling the treatment times does not change the probabilities of treatment success. In agreement with our previous results, treating with BDF is a better strategy; it corresponds to a finite number of cycles. If treating with WDF, the optimum corresponds to an "infinite" number of cycles. In Fig. 5(b) we decrease the treatment time by approximately a factor of 3 . The effect is very noticeable. First of all, we can see that the maximum probability of treatment success achieved by shorter protocols is significantly lower than that for longer protocols. Further, we observe that treating with BDF now has a larger advantage compared to treating with WDF. Also, the contour plot of the probabilities changes its shape significantly: for BDF treatments, the optimal treatment consists of only 2 cycles of each of the drugs, and for WDF treatments-of 3 cycles.

Figure 6 demonstrates the effects of decreasing the total treatment time in a systematic way. As $T_{\text {treat }}$ decreases, the probability of treatment success decreases (Fig. 6(a)), and the difference between the optimal BDF and WDF treatment protocols increases. The BDF strategy remains advantageous. The optimal number of cycles (Fig. 6(b)) decreases as the treatment time decreases. The optimal BDF protocol usually requires fewer cycles than the optimal WDF protocol. Very short treatment times require the optimal protocol to have only one cycle of drug application. For 
(a)

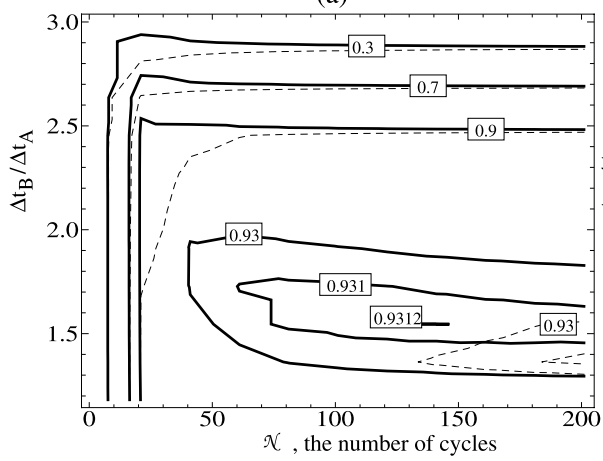

(b)

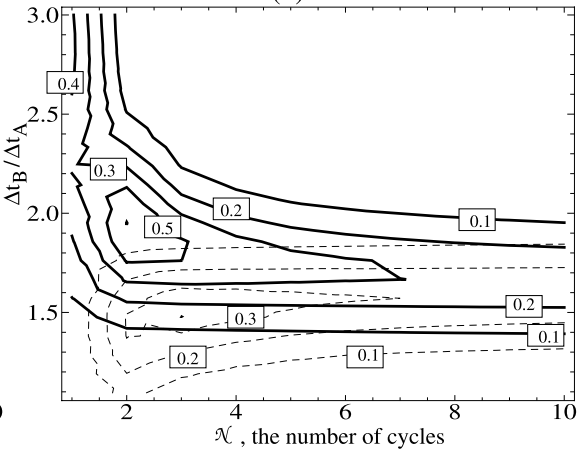

Fig. 5 Drugs of different potencies in protocols of long and short durations. Drug $A$ has $h=5$, drug $B$ has $h=3$. (a) The treatment time is $T_{\text {treat }}=74.83$, (b) $T_{\text {treat }}=25$. The other parameters are $l=1, d=0$, $u=10^{-8}, N=10^{13}$. Solid lines correspond to BDF strategies, and dashed lines to WDF strategies

(a)

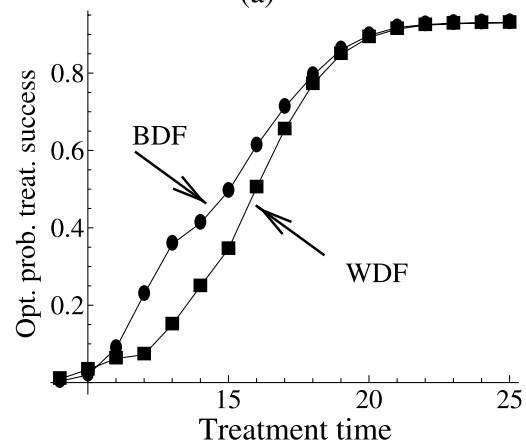

(b)

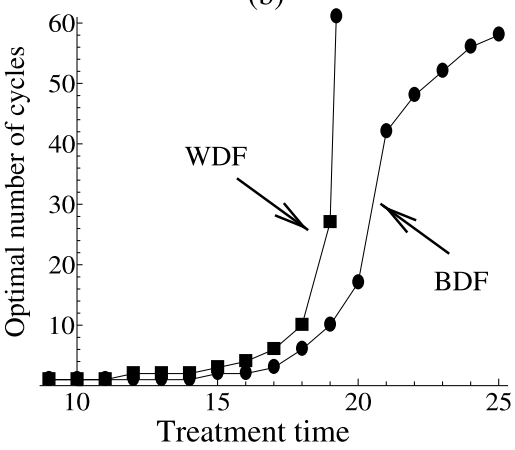

Fig. 6 Drugs of different potencies in protocols of different durations. Drug $A$ has $h=5$, drug $B$ has $h=3$. (a) The optimal probability of treatment success as a function of treatment time. (b) The optimal number of cycles as a function of treatment time. The graphs are presented for the WDF and BDF strategies. The other parameters are $l=1, d=0, u=10^{-8}, N=10^{13}$

BDF treatments, the probability of treatment success experiences a slowing down in its growth as a function of $T_{\text {treat }}$. This corresponds to the regime where having only one cycle is no longer optimal, and the optimal strategy requires using more than one cycles.

Next we will assume that the two drugs have an equal potency and only differ by their activity spectra. In Fig. 7, we consider two drugs with highly different mutation rates, drug $A$ with $u=10^{-5}$ and drug $B$ with $u=10^{-9}$. In Fig. 7(a), we fix the treatment time to be approximately 16.49 time units, which for the parameter values chosen is comparable with the time it takes on average to eliminate the colony of susceptible cells with one of the drugs (approximately 12.66 units). The contour plots of the probability of treatment success obtained by the stochastic method are presented for the two cases: $\operatorname{drug} A$ first (solid lines) and drug $B$ first (dashed lines). We observe that the optimal probabilities of treatment success in the two scenarios 
(a)

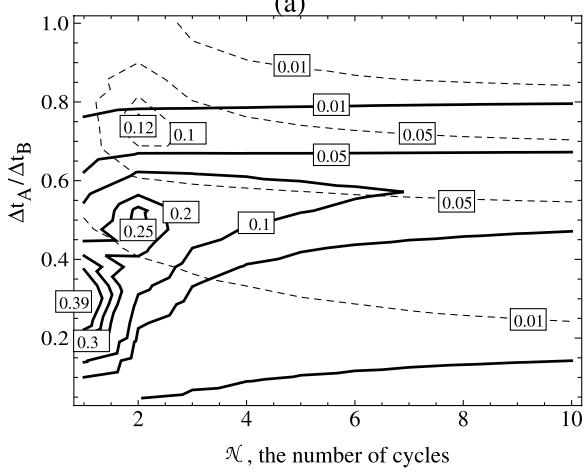

(b)

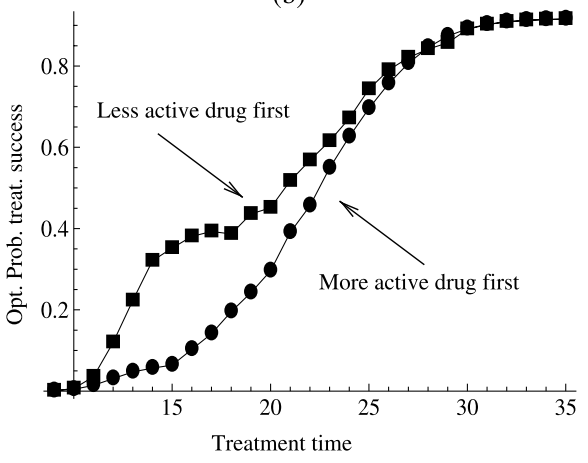

Fig. 7 Drugs of different activity spectra in protocols of different duration. Drug $A$ has $u=10^{-5}$, drug $B$ has $u=10^{-9}$. (a) The contour plots of the probability of treatment success for treatment time $T_{\text {treat }}=16.46$; solid lines correspond to $u_{1}>u_{2}$ (drug $A$ first), and dashed lines to $u_{1}<u_{2}$ (drug $B$ first). (b) The optimal probability of treatment success as a function of treatment time. The other parameters are $l=1, d=0, h_{1}=h_{2}=3, N=10^{11}$

differ significantly. If we start with drug $B$, the optimal strategy is to use 2 cycles, and the corresponding success probability is about 0.12 . If, on the other hand, we start treatment with drug $A$, then the best strategy is to use only one cycle, and the corresponding success probability is about 0.39 , which is significantly higher. In both cases, the drug with the lower mutation rate must be used for longer.

In Fig. 7(b), we show that for most finite treatments protocols, it is advantageous to start with the drug characterized by a higher mutation rate, but use the other (more active) drug for longer cycle durations. The difference between the two types of protocols decreases as the treatment length increases, and for very long treatments, the two lines in Fig. 7(b) cross over. As was described in the previous section, in the limit of long treatments, it becomes slightly advantageous to treat with the broader drug first.

We can see that while the deterministic model works well for infinite treatments, it fails to explain the observations for short treatments. Incorporating the finite treatment time effects (27) does not help explaining why as the total treatment length becomes shorter, using only one or two cycles becomes the optimal strategy. The intuition behind this is as follows.

If the treatment time is long, the extinction of the susceptible colony as well as both partially-resistant colonies is a certainty, and the probability of treatment success is defined by the dynamics of partially-resistant mutants, which are well described by the deterministic model. On the other hand, the dynamics of drug resistance for short treatment times has a purely stochastic component. If the treatment time is short, the probability of treatment success largely depends on the chance of the extinction of the susceptible and the partially susceptible colonies by the end of the treatment. For example, if the time $T_{\text {treat }}$ is so short that even the elimination of the fully-susceptible colony is unlikely, then the probability of treatment success is extremely low. This corresponds to the regime in Figs. 5(a) and 7(b) with treatment time below approximately 9 ; a rough estimate for this minimum treatment time which leads to clinically 
meaningful treatment probabilities is $\ln N /(h+d-l)$. For treatment times longer that this threshold, the main reason for treatment failure becomes the non-extinction of partially resistant mutants. While the fully-susceptible colony is killed by the drugs continuously, the two partially-resistant colonies are killed intermittently. The optimal treatment protocol maximizes the chances of eliminating these colonies by the end of the treatment.

\section{Protocols Involving Drugs of High Toxicity}

So far we assumed that the objective for treatment protocol optimization is to maximize the number of cancer cells killed. This is a reasonable strategy given that the drugs' side effects are relatively mild. The question of optimization has to be approached differently for treatments with drugs of high toxicity. In this context, the optimization problem has two objectives: (1) maximize the probability of cancer eradication, and (2) minimize the side effects of the treatment.

The framework developed in this paper is applicable in such situations, but the rules (such as the BDF rule) derived without considerations of toxicity, may not hold. In what follows we describe how to apply our framework to drugs of high toxicity.

By optimizing the probability of cancer eradication, we can produce contour plots such as the ones in Fig. 3. Let us consider a particular example where two drugs are used: $\operatorname{drug} A$ has potency $h_{A}=5$ and drug 2 has potency $h_{B}=3$. In Fig. 8 we present the contour plots of the objective function $F /\left(l N \log N u_{1} u_{2}\right)$, with the axes $\Delta t_{B}$ (the cycle duration of the worse drug) and $\Delta t_{A} / \Delta t_{B}$. Note that for the purposes of this section it is more convenient to use the duration of one of the drugs' cycle (drug $B$ ) as the horizontal axis. This is different from Fig. 3 where the horizontal axis was the cycle duration of the first drug used. Treatment starting with the best drug corresponds to the solid contours, and treatment with the worst drug first- to
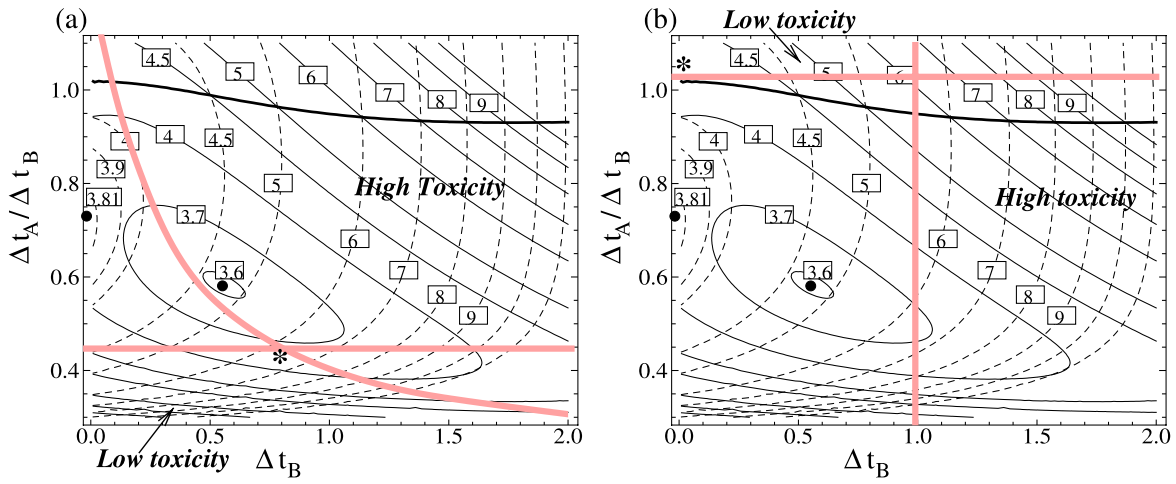

Fig. 8 Contour plots of the objective function for the BDF (solid lines) and the WDF (dashed lines) strategies, for drugs of high toxicity. The axes are $\Delta t_{B}$ (the cycle duration of the worse drug) and $\Delta t_{A} / \Delta t_{B}$ (the ratio of the best-to-worst cycle durations). (a) Drug $A$ is more toxic, (b) drug $B$ is more toxic. The unrestricted optima for BDF and WDF strategies are marked by black dots; the optima under the toxicity assumptions are marked by stars. The parameters are: $l=1, d=0, h_{A}=5, h_{B}=3, u_{1}=u_{2}=10^{-5}$, $u_{12}=100 u_{1} u_{2}, N=10^{10}$ 
dashed contours. Without considerations of toxicity, we simply find the minima of the functions plotted (recall that it is the minimum of function $F$ that optimizes treatment in the deterministic approach). For the BDF and the WDF scenarios these minima are indicated by black dots in the figure. The BDF treatment (solid contours) has a lower minimum, and the optimum strategy requires using $\operatorname{drug} B$ for duration $\Delta t_{B} \approx 0.55$ and drug $A$ for duration $\Delta t_{A} \approx 0.40$. The percentages of time when drugs $A$ and $B$ are administered are given by $42 \%$ and $58 \%$ respectively.

To prepare for further optimization restrictions coming from the drug toxicity, in Fig. 8 we compared the two types of treatments (BDF and WDF) for each point $\left(\Delta t_{A}, \Delta t_{B}\right)$. The thick black line near the top of the contour plot separates the region where the BDF protocols are better (below the line), from the region where WDF protocols are better (above the line). The line corresponds to the points where the functions $F /\left(l N \log N u_{1} u_{2}\right)$ calculated for the two regimes are equal to each other (we can see that the same-level, solid and dashed, contours intersect on that line). If for some reason toxicity restrictions require us to use protocols corresponding to the cycle lengths above this line, then a WDF treatment will be preferable.

There are several ways in which the optimization problem can be formulated in the presence of drug side effects. One way is to assign different weights to different optimization goals, and formulate the new objective function to be minimized. For example, the new objective function can be the weighted sum of the function $F$ discussed before, and another function representing the side effects, which is an increasing function of cycle durations and/or percentages of time a drug is used. The difficulty with this approach is a certain arbitrariness of the choice of the weighting coefficients. For this reason, here we adopt a simpler approach. We will identify all possible protocols which are acceptable from the point of view of side effects. This is equivalent to separating the $\left(\Delta t_{A}, \Delta t_{B}\right)$ space into regions of high and low toxicity. Then, we will perform the minimization of the original objective function restricted to the region of low toxicity. Below we consider two examples of application of this method (Fig. 8(b,c)).

First, let us assume that drug $A$ (the stronger of the drugs) is very toxic. To minimize side effects experienced by the patient, one must minimize (i) the cycle lengths of $\operatorname{drug} A$, and (ii) the percentage of time that $\operatorname{drug} A$ is administered. Let us denote $v=\Delta t_{A} / \Delta t_{B}$ (the vertical axes in Fig. 8). The above two requirements can be written as follows:

(i) $\Delta t_{A}<c_{1}$, where $c_{1}$ is some constant. This inequality can be rewritten as $v<$ $c_{1} / \Delta t_{B}$, and corresponds to the region below the hyperbola in Fig. 8(a).

(ii) The percentage of time that $\operatorname{drug} A$ is administered is given by $v /(1+v)$. Requirement $v /(1+v)<c_{2}$, where $c_{2}<1$ is some constant, leads to the inequality $v<c_{2} /\left(1-c_{2}\right)$. This corresponds to the region below the straight horizontal line in Fig. 8(a).

From the above considerations, the relatively low toxicity region corresponds to the bottom left corner in Fig. 8(a). Obviously, the protocol previously identified as optimal cannot be used, and the optimization procedure must happen within the low toxicity region. Since this region is below the thick black line, a BDF protocol will still be the strategy of choice. Minimizing the function $F$ under these constraints, 
we find the new optimum, which is indicated by a star in Fig. 8(a). It corresponds to $\Delta t_{A}=0.36$ and $\Delta t_{B}=0.8$; the percentages of time when drugs $A$ and $B$ are administered are given by $31 \%$ and $69 \%$ respectively. As we can see, the new optimum treatment has a shorter cycle duration for $\operatorname{drug} A$ and a lower percentage of time when it is used, compared to the old, unrestricted optimum.

In the second example, let us assume that drug $B$ is more toxic than $\operatorname{drug} A$. This imposes the following requirements: (i) the cycle lengths of drug $B$ should be minimized, and (ii) the percentage of time that drug $B$ is administered should be restricted. In our notations, this leads to the following restrictions:

(i) $\Delta t_{B}<c_{3}$, where $c_{3}$ is some constant. This corresponds to the region to the left of a straight vertical line in Fig. 8(b).

(ii) The percentage of time that drug $B$ is administered is given by $1 /(1+v)$. Requirement $1 /(1+v)<c_{4}$, where $c_{4}<1$ is some constant, leads to the inequality $v>\left(1-c_{4}\right) / c_{4}$. This corresponds to the region above the straight horizontal line in Fig. 8(b).

It follows that the relatively low toxicity region corresponds to the top left corner in Fig. 8(b). Now this region is above the thick black line, and therefore a WDF strategy is preferable. The new optimum is indicated by a star in Fig. 8(b), and corresponds to short (mathematically, zero-length) cycles of both drugs, such that the percentage of time when drug $B$ is administered is below $50 \%$, which is lower than the $58 \%$ of the original optimum.

The examples above describe the following general trend. If the best drug is also more toxic, then the optimal protocol under the toxicity restrictions still starts with the best drug (but uses it for a shorter cycle duration, and at a smaller percentage of time). If however the worst drug is more toxic, then the optimal treatment strategy may start with the worst drug. This is the only case we found where the BDF rule could be overturned. In this case, the better drug must be used for relatively longer durations, and the total cycle durations must be kept short.

To explain this we note that both BDF and WDF optimal strategies require a longer cycle duration for the worst drug; however, the ratio of the best to worst cycle length is smaller in the BDF case. If the best drug is more toxic, we impose a requirement that it should be used at a smaller percentage of time; the corresponding region of the parameter space (the lower left corner) is closer to the BDF optimum, and thus will require a BDF strategy. On the other hand, if the worst drug is more toxic, the low toxicity subspace corresponds to the upper left corner, and may happen in the region where WDF strategies are optimal. For more details on the theoretical aspects of the objective function shape, we refer the reader to Appendix A.

\section{Discussion and Conclusions}

In this paper we studied cyclic drug therapies with the aim to develop general guidelines on optimal treatment scheduling. Our work continues earlier studies of Day (1986b) and extends the results to cross-resistant drugs.

The main idea behind treatment with multiple drugs is as follows. One of major causes of cancer drug treatment failure is the development of drug resistance, which 
is often associated with genetic events that modify cellular phenotype inside the tumor. Drug resistance can potentially be overcome by the combination of multiple drugs, where a mutation that confers resistance against one drug does not confer resistance against any of the other drugs in use. Cyclic drug therapies consist of several alternating treatment courses, such that each of the drugs gradually eradicates the population of susceptible cells, and the net effect of the alternating cycles of treatment is the decline of the tumor. We find that in order for a cyclic treatment to be feasible, the drugs' strengths must satisfy a certain condition, which we call the condition of "mutual strength." Our results suggests that for mutually strong drugs, the success of cyclic treatments depends on the exact scheduling, and in particular, on the following factors: (i) which drug is administered first, and (ii) the durations of the treatment courses.

The methodology used in this paper can be applied directly to cancer drugs with relatively mild side effects, such as small molecule kinase inhibitors (Zhang et al. 2009). Kinases are currently one of the most promising and most intensively pursued drug targets (Arkin and Wells 2004), because of their high efficiency and low toxicity. One example is Imatinib (O’Dwyer et al. 2002; Deininger and Druker 2003), the first selective tyrosine kinase inhibitor targeting Bcr-Abl protein, which has shown clinical efficacy in the treatment of Chronic Myeloid Leukemia (CML). Small molecule inhibitors such as Imatinib, have the ability to bind specifically to cancer cells, and spare healthy, non-cancerous cells. Currently, there are 11 kinase inhibitors that have received US Food and Drug Administration approval as cancer treatments, and there are many more that are at different stages of development (Zhang et al. 2009).

Because of relatively mild side effects of these drugs, the main objective of protocol optimization is a maximal efficiency in killing cancer cells. In this context, our main findings in the absence of cross-resistance can be summarized as follows:

- For a cyclic therapy to be effective, the two drugs must be mutually strong (condition (33)).

- Variant (a) of the worst drug rule of Day (1986b) ("use more of the worst drug") is confirmed for all scenarios in the absence of cross-resistance. For each parameter combination, we can find the most successful cyclic strategy, characterized by the optimal cycle durations for both drugs. The duration for the worse drug is always longer.

- Variant (b) of the worst drug rule ("use the worst drug early") does not hold in general. In other words, the optimal cyclic strategy usually starts with the better of the drugs.

- If the two drugs have equal strengths, but different activity spectra, then the best strategy depends on the length of treatment. For very long treatments that ensure a complete eradication of all the susceptible mutants, the best protocol starts with the drug with a broader activity spectrum. Note however that the relative gain in starting with the "best" drug compared with starting with the "worst" drugs is very small in this situation. For suboptimal treatment durations it becomes advantageous to start treatment with the narrower drug. In both scenarios, the optimal cycle duration of the narrower drug is shorter than that of the broader drug, so in this sense, we have a "use less of the worst drug" rule in place. 
Most chemotherapeutic agents used to treat cancer are not as selective as small molecule inhibitors at killing cancerous cells. Their efficiency against cancerous cells has a side effect of a high killing rate of healthy cells, and thus a prolonged administration of such drugs cannot be tolerated by patients. Our methodology can also be applied, with some modifications, to drugs of high toxicity. We find that unless the worst drug is also more toxic than the better drug, the "best drug first, worst drug for longer" rule holds even in the presence of side effects. The parameters for the optimal protocol can be calculated by the method described in Sect. 7, and will differ from the optimal protocols for drugs with relatively mild side effects.

In the next section we continue our discussion of small molecule inhibitors and summarize the role of cross-resistance for optimal treatment design. The above remarks about toxic drugs hold true in the presence of cross-resistance.

\subsection{The Role of Cross-Resistance}

Currently there are three small molecule inhibitors approved for treatment of CML: Imatinib (O'Dwyer et al. 2002; Deininger and Druker 2003), Dasatinib, and Nilotinib (Bradeen et al. 2006; Weisberg et al. 2007). These drugs are all inhibitors of the BCR-ABL gene product. It has been shown that these three drugs are cross-resistant because of one mutation (T315I) which confers resistance against all those drugs. In addition to this one mutation, more than 50 mutations have been identified that confer resistance against one of the drugs (in particular Imatinib), but not against the others (Deininger 2007; Quints-Cardama et al. 2007). Given this situation, the question arises whether a cyclic therapy with cross-resistant drugs can be optimized to produce the best results.

We found that in the presence of cross-resistance, the tendency is that the better drug (the drug with a stronger potency) must be used first and for longer periods of time. This reinforces our previous result that the optimal strategy should start with the better drug. However, this tendency is in conflict with the rule that the worst drug should be used for longer periods of time. The trade-off works out to be the following: if we start with the better (stronger) drug, then, in the presence of cross-resistance, the cycles for the worse drug should still be longer than those for the better drug. However, if we start with the worse drug, the duration of the cycles for the better drug should be longer.

When we are dealing with drugs of similar potency but different activity spectra, the presence of cross-resistance does not change the results qualitatively, but it makes the effect observed in the absence of cross-resistance weaker. In other words, the optimal strategy is still to start with a broader activity drugs and have its cycles longer than those for the narrower drug, but the difference between the success rate of the optimal strategy and other, suboptimal strategies, is small.

\subsection{Testing the Theory}

Since the publication of the papers by Goldie and Coldman (1979, 1983b, 1998), Goldie et al. (1982), Coldman and Goldie (1985) and Day (1986b), there have been several attempts by clinical oncologists to verify the theoretical results, and in particular, the famous "worst drug rule." Several papers concluded that the worst drug 


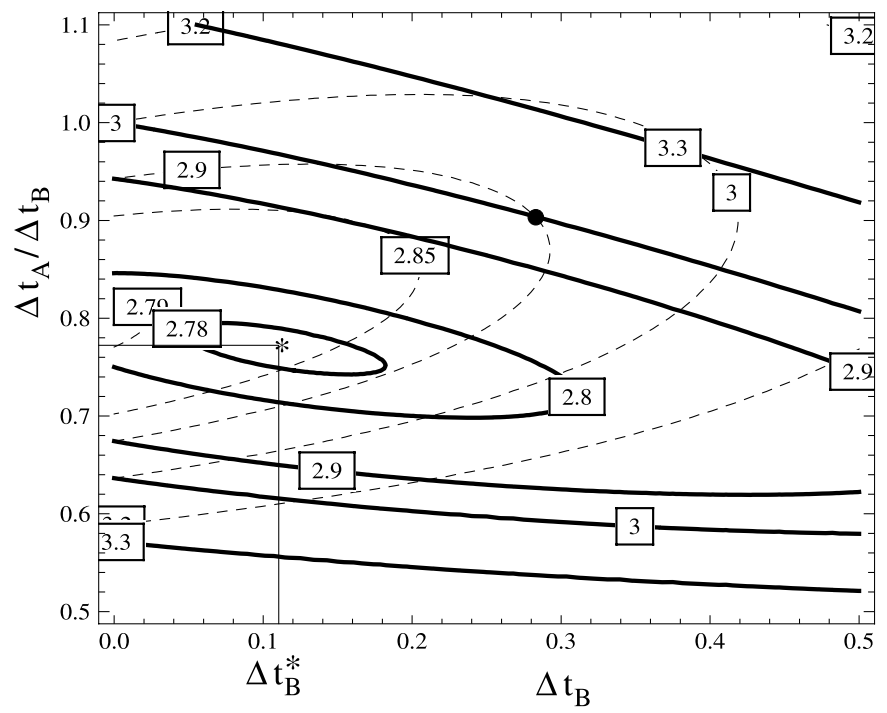

Fig. 9 Same as Fig. 3(a); the BDF (thick solid lines) and the WDF (dashed lines) strategies are superimposed, and the axes are $\Delta t_{B}$ (the cycle duration of the worse drug) and $\Delta t_{A} / \Delta t_{B}$ (the ratio of the best-to-worst cycle durations). The values of function $F_{1 \rightarrow 2}((28)$, scaled) are marked next to the contours. Lower numbers correspond to more successful strategies

rule does not hold, see papers Colucci et al. (1997), Gebbia et al. (2003), Fulfaro et al. (2003), Grossi et al. (2007) in the context of non-small-cell lung cancer. These results may seem discouraging, but a careful examination of the methods reveals that a more subtle approach must be used for experimental verification/rejection of the theoretical predictions. Let us consider the following example. Suppose the theory predicts that the optimal strategy for cyclic treatments with drugs $A$ and $B$ starts with drug $A$ and requires cycle durations $\Delta t_{A}^{*}$ and $\Delta t_{B}^{*}$; see Fig. 9 for illustration. Let us consider two treatment strategies where $\operatorname{drugs} A$ and $B$ are alternated with cycle durations $\Delta t_{A} \neq \Delta t_{A}^{*}$ and $\Delta t_{B} \neq \Delta t_{B}^{*}$ respectively (in other words, the cycle durations are chosen to be suboptimal; in Fig. 9 this corresponds to the black dot with $\Delta t_{B} \approx 0.28$ and $\left.\Delta t_{A} / \Delta t_{B} \approx 0.9\right)$. An experiment can be set up where strategy $A B A B A B A B$ is compared with strategy $B A B A B A B A$ with the given values for cycle durations. It is possible that out of these two strategies the one starting with drug $B$ leads to a better treatment success rate (in Fig. 9 we can see that the black dot belongs to the 3.0 contour of the best-drug-first strategy, and the 2.9 contour of the worst-drug-first strategy, suggesting that it is better to use the worst drug first). An outcome of this kind is consistent with the theory, but at the same time it may lead to a wrong conclusion that the theory is wrong, because the theoretical optimal treatment starts with drug $A$ (and corresponds to the star in Fig. 9), and the experimentally found best treatment (out of the two suboptimal treatment strategies) starts with drug $B$.

This simple example illustrates the point that in order to apply the theory developed here, one needs to have more information about the system parameters. In particular, the knowledge of the drugs' potencies and activity spectra, as well as the rate 
at which cross-resistant mutants are generated, can help optimizing cyclic treatment strategies.

In general, the methodology developed here can help creating more detailed theories of cyclic drug treatments with non-symmetric rates, and different activities and potencies of the two drugs. It can also be extended to more than two drugs. Another important extension is to create a complete theory of short (suboptimal) treatments in the context of toxic drugs. The objective is to optimize the protocol which has to be kept short because of drugs' side effects. This is a subject of current research.

Acknowledgements N.K. gratefully acknowledges the support of the Sloan Fellowship and the NIH grants: R01 CA129286 and R01 AI-58153.

Open Access This article is distributed under the terms of the Creative Commons Attribution Noncommercial License which permits any noncommercial use, distribution, and reproduction in any medium, provided the original author(s) and source are credited.

\section{Appendix A: Some Properties of the Objective Function}

Let us refer to the two drugs used as $\operatorname{drug} A$ and drug $B$. We suppose that they have potencies $h_{A}$ and $h_{B}$, and cycle durations $\Delta t_{A}$ and $\Delta t_{B}$. Further we assume that drug $A$ is stronger: $h_{A}>h_{B}$. In order to find the best treatment protocol, we need to evaluate the objective function $F$ (28)-(30), and find the minimum over the space $\left(\Delta t_{A}, \Delta t_{B}\right)$. The function $F$ depends on parameters $h_{1}, h_{2}, \Delta t_{1}, \Delta t_{2}$. The BDF and the WDF treatments can be parameterized according to Table 1 . The objective functions corresponding to the two regimes are denoted as $F_{\mathrm{WDF}}$ and $F_{\mathrm{BDF}}$. Let us denote by $v$ the ratio $v=\Delta t_{A} / \Delta t_{B}$. It is possible to prove that

$$
F_{\mathrm{WDF}}\left(\Delta t_{B}, v\right)=F_{\mathrm{BDF}}\left(-\Delta t_{B}, v\right) \text {. }
$$

The reason for this symmetry is that the initial-value problem (25)-(26) is invariant under the transformation $h_{1} \leftrightarrow h_{2}, \Delta t_{1} \leftrightarrow \Delta t_{2}, t \rightarrow-t$. This statement remains true even if we include cross-resistance. One immediate consequence of (34) is that $F_{\mathrm{WDF}}(0, v)=F_{\mathrm{BDF}}(0, v)$; in other words, in the limit of a zero cycle duration, it does not matter what drug we start the treatment with. Another consequence of symmetry property (34) is that the function $F_{\mathrm{WDF}}\left(\Delta t_{B}, v\right)$ for $-\infty<\Delta t_{B}<\infty$ and $\frac{1}{h_{A}-1}<v<h_{B}-1$ comprises all the information about the minima of the objective function, where positive values of $\Delta t_{B}$ correspond to WDF protocols, and the negative values - to BDF protocols. Thus, the optimization task is reduced to finding the minimum of only one function, $F_{\mathrm{WDF}}$. If this minimum happens at a negative value of $\Delta t_{B}$, then a BDF strategy is optimal.

Table 1 Best drug first and worst drug first treatments

\begin{tabular}{llcccc}
\hline Treatment type & Objective function notation & $h_{1}$ & $h_{2}$ & $\Delta t_{1}$ & $\Delta t_{2}$ \\
\hline WDF & $F_{\mathrm{WDF}}$ & $h_{B}$ & $h_{A}$ & $\Delta t_{B}$ & $\Delta t_{A}$ \\
BDF & $F_{\mathrm{BDF}}$ & $h_{A}$ & $h_{B}$ & $\Delta t_{A}$ & $\Delta t_{B}$ \\
\hline
\end{tabular}


We observe that the function $F_{\mathrm{WDF}}\left(\Delta t_{B}, v\right)$ always has a unique, global minimum corresponding to a negative value of $\Delta t_{B}$. This means that the optimal strategy is a BDF treatment. The corresponding optimal cycle duration, $\left|\Delta t_{B}\right|$, grows with the difference in drug potencies, $h_{A}-h_{B}$. The corresponding optimal value of $v=\Delta t_{A} / \Delta t_{B}$ is always less than 1 , decreasing with the difference $h_{A}-h_{B}$. In other words, for very different drugs, the cycle length of the worse drug is larger, and also the ratio of the best-to-worst cycle length is smaller. The optimum duration $\Delta t_{B}$ also increases with the amount of cross-resistance.

From the fact that there is only one global minimum of this function which is situated in the left half-plane, it follows that for WDF treatments, the optimum occurs on the domain boundary (that is, it corresponds to $\Delta t_{B}=0$, because the value of the objective function for $v=\frac{1}{h_{A}-1}$ and $v=h_{B}-1$ is infinity).

If we restrict the values of $\Delta t_{B}$ to the positive half-plane (WDF treatments) we observe that the optimum treatment corresponds to the higher ratios, $v$, compared to those for the global minimum (BDF treatments). This helps explaining the general trend described in Sect. 7, where we find that under the restrictions of toxicity, the best protocol starts with the stronger drug, if this drug is more toxic, and it may start from the weaker drug if the weaker drug is more toxic.

\section{Appendix B: Treatment with Drugs that Are Not Mutually Strong}

Let us suppose that the drugs that are used in a cyclic therapy fail condition (33). There are three cases (see also Fig. 2):

(a) $h_{1}<\gamma$ and $h_{2}<\gamma$ (region F in Fig. 2);

(b) $h_{1}<\gamma$ or $h_{2}<\gamma$, but not both (regions D and E in Fig. 2);

(c) $h_{1}>0, h_{2}>0$, but $h_{1}^{-1}+h_{2}^{-1}>\gamma^{-1}$ (region C in Fig. 2).

A qualitative description of the system behavior in these regions is presented in Sect. 4. Here we present some analytical considerations regarding case (c).

In case (c), let us suppose that $h_{1}>h_{2}$. The best treatment strategy is this case is the following. Start from the stronger of the drugs and try to eliminate the mutants susceptible for that drug. Then switch to the second drug and treat indefinitely. A strategy of this type is characterized by the duration of the first cycle, $\Delta t_{1}$. We can show that this quantity has an optimum. The probability of treatment failure in this case has the following two main contributions: (i) the probability to still have mutants of type $x_{01}$ after the time $\Delta t_{1}$ from the start of treatment, and (b) the probability to produce double-hit mutants out of type $x_{10}$-mutants (the probability of producing double-hit mutants out of the $x_{01}$-type mutants is much smaller than contribution (i)).

Contribution (i) can be calculated analytically by solving the characteristic equation $\dot{x}=l x^{2}-\left(l+d+h_{1}\right) x+\left(d+h_{1}\right)$, where $h_{1}$ is assumed to be the stronger drug, and the initial condition is $x(0)=0$. The probability of having mutants of type $x_{01}$ after time $\Delta t_{1}$ is given by $1-x\left(\Delta t_{1}\right)^{N_{1}}$, where $N_{1}$ is the initial abundance of type $x_{01}$-mutants. This function decays with $\Delta t_{1}$. This is intuitively clear: the longer we treat with drug 1 , the higher is the chance to get rid of mutants $x_{01}$.

Contribution (ii) can be calculated by a similar method, assuming that for duration $\Delta t_{1}$, the colony of type $x_{10}$-mutants grows stochastically with rate $\gamma$, and after that 
(a)

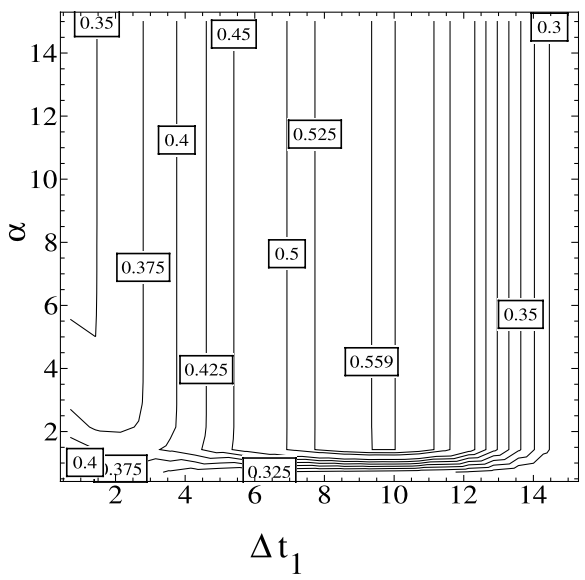

(b)

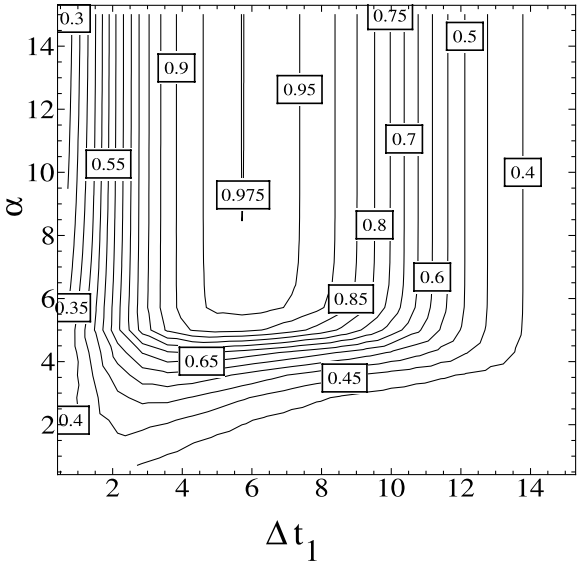

Fig. 10 Treatment with weak drugs. The probabilities of treatment success calculated numerically by the stochastic method, are presented as contour plots. The axes are $\Delta t_{1}$ and $\alpha$. (a) The worst drug first, $h_{1}=1.2, h_{2}=2.2 ;(\mathbf{b})$ The best drug first, $h_{1}=2.2, h_{1}=1.2$. The rest of the parameters are $l=1, d=0$, $u_{1,2}=10^{-6}, N=10^{6}$

it decays indefinitely, with average rate $\gamma-h_{2}$. An analytical solution cannot be obtained in this case, but it is clear that the probability of treatment failure due to $x_{11}$ production by $x_{10}$ is a growing function of $\Delta t_{1}$. This is because the longer we treat with the first drug, the longer growth phase is experienced by the $x_{10}$-mutants, and the higher is the chance that they produce fully-resistant mutations.

It is apparent that we have a trade-off between two failure mechanisms. It turns out that an intermediate optimal value of $\Delta t_{1}$ maximizes treatment success probability in this case. Figure 10 illustrates these findings. We present a contour plot of the probability of treatment success (calculated numerically by using the stochastic methodology), as a function of parameters $\Delta t_{1}$ and $\alpha$. We can see that there is an optimal (and comparatively large) value of the first cycle duration, $\Delta t_{1}$; the optimal values of $\alpha$ belong to an interval $\alpha>\alpha_{*}$, for some $\alpha_{*}>1$. We observe that for these values, treating for more than two cycles does not change the probability of treatment success.

As $h_{2}$ increases, it may become more efficient to return to treating with the first drug. In this case, several rounds of the strategy described above are applied. Even though, on average, one of the partially-resistant mutants (namely, $x_{01}$ treated by the first drug) is expected to grow, this strategy maximizes the chances of its extinction by driving it periodically to low numbers. This corresponds to an appearance of another maximum in the contour plot of Fig. 10 (not shown). This maximum (which corresponds to values $\alpha<1$ and relatively small values of $\Delta t_{1}$ ) grows with $h_{2}$ while the maximum corresponding to the twocycle treatment becomes less pronounced (not shown). The idea is that the expected growth is slow, such that at the end of each round, the average gain is small. 


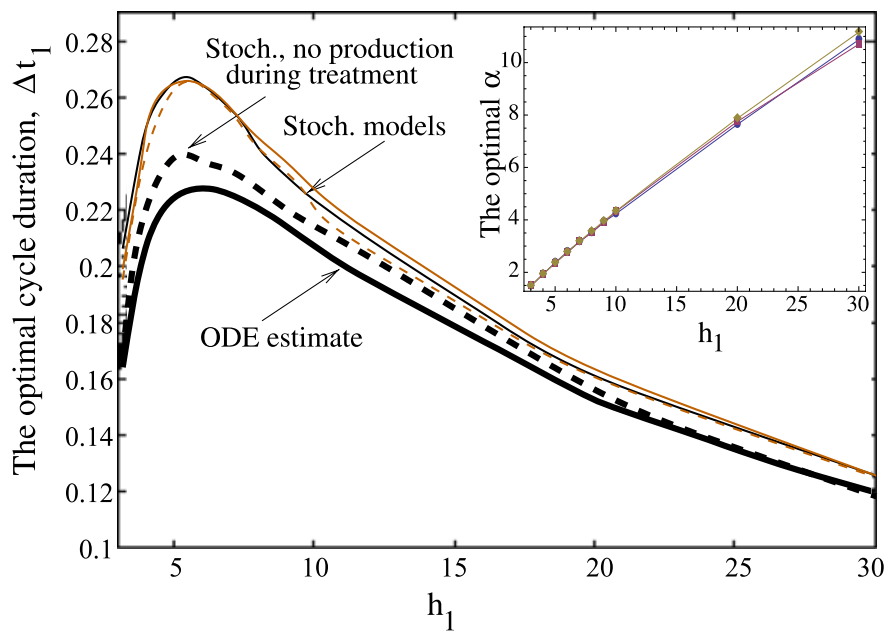

Fig. 11 The optimal cycle duration, $\Delta t_{1}$, as a function of $h_{1}$ (with fixed $h_{2}=2$ ), calculated by different methods. The thick solid line corresponds to the ODE model $(l=1, d=0)$; the thick dashed line-to the stochastic model with no mutant production $\left(l=1, d=0, u=10^{-6}\right)$. The three thin lines correspond to the full production stochastic model with $l=1, d=0, u=10^{-6}$ (small dash); $l=5, d=4, u=10^{-6}$ (solid); $l=1, d=0, u=10^{-5}$ (long dash). Inset: the optimal values of $\alpha$ calculated by the full production stochastic model (triangles), the stochastic model with no production (squares) and the ODE estimate (diamonds); in all cases $l=1, d=0, u=10^{-6}$. The tumor size is $N=10^{10.04}$

\section{Appendix C: Comparison of the Deterministic and Stochastic Predictions}

The results in Fig. 3 were obtained by using the deterministic approximation of Sect. 3. The question is whether this method gives a good agreement with stochastic simulations of Sect. 2. The comparison of the optimal values of $\Delta t_{1}$ and $\alpha$ calculated by the deterministic and stochastic model is presented in Fig. 11. There, we fix the value $h_{2}$ and plot the optimal value $\Delta t_{1}$ as a function of $h_{1}$ (the plot of the optimal value $\alpha$ is shown in the inset).

We can see that while the deterministic approximation predicts the optimal $\alpha$ values extremely well, the calculated optimal cycle duration, $\Delta t_{1}$, is slightly lower than that obtained by the stochastic model. One of the reasons is that in the ODE model, we ignored the production of partially resistant mutants during treatment. The thick dashed line in Fig. 11 corresponds to a stochastic calculation where we "turned off" the single-hit mutants after the start of treatment. The agreement between the modified stochastic and the deterministic model is better. The remaining difference is attributable to the purely stochastic effects which are not captured by the equations for the averages; see also Appendix D.

\section{Appendix D: Equal Treatment Strength, Different Mutation Rates}

Suppose at the start of treatment the number of mutants resistant to drug $1, x_{10}$, equals $N_{10}$, and the number of mutants resistant to drug $2, x_{01}$, equals $N_{01}$. Let us calculate 
the probability that no fully-resistant mutants are produced by time $t$ under a one-drug treatment scenario.

To solve this problem, we will need to consider the following equations for characteristics:

$$
\begin{aligned}
\dot{\xi}_{1} & =l(1-u) \xi_{1}^{2}+\left(l u \xi_{2}-(l+d+h)\right) \xi_{1}+d+h, \\
\dot{\xi}_{2} & =l \xi_{2}^{2}-(l+d) \xi_{2}+d, \\
\xi_{1}(0) & =1, \\
\xi_{2}(0) & =0 .
\end{aligned}
$$

These equations correspond to the situation where double-hit mutants are produced at a mutation rate $u$ from one-hit mutants, and the effect of treatment on one-hit mutants is expressed by $h$. The probability of non-production of double-mutants is given by

$$
P\left(u, h, N_{1} ; t\right)=\xi_{1}(u, h ; t)^{N_{1}},
$$

where $N_{1}$ is the initial abundance of one-hit mutants, and 0 is the initial abundance of double-hit mutants. The solution can be found exactly if we set $d=0$. In this case, $\xi_{2}(t)=0$, and (35) is a Riccati equation with solution

$$
\xi_{1}(u, h ; t)=-\frac{\beta_{1} A e^{\beta_{1} t}+\beta_{2} e^{\beta_{2} t}}{l(1-u)\left(A e^{\beta_{1} t}+e^{\beta_{2} t}\right)},
$$

where

$$
\begin{aligned}
\beta_{1,2} & =\frac{1}{2}\left(-(l+h) \pm \sqrt{(l+h)^{2}-4 h l(1-u)}\right), \\
A & =-\frac{l(1-u)+\beta_{2}}{l(1-u)+\beta_{1}} .
\end{aligned}
$$

In particular, for $h=0$, the calculations simplify to give

$$
\xi_{1}(u, 0 ; t)=\frac{1}{1-u\left(1-e^{l t}\right)} .
$$

Let us suppose that drug 1 is used for treatment. Then the probability that fullyresistant mutants are not produced is given by

$$
P_{1}=P\left(u_{2}, 0, N_{10}\right) \times P\left(u_{1}, h, N_{01}\right) .
$$

Similarly, under drug 2 treatment we have

$$
P_{2}=P\left(u_{2}, h, N_{10}\right) \times P\left(u_{1}, 0, N_{10}\right) .
$$

For the initial mutant abundances we can take the expected values:

$$
N_{10}=u_{1} N \log N, \quad N_{01}=u_{2} N \log N,
$$


where $N$ is the tumor size at the start of treatment.

It turns out that if $u_{1}<u_{2}$, then $P_{1}>P_{2}$, that is, the probability of double-mutant non-production is higher if we start treatment with the drug with the lower mutation rate.

It is instructive to see that if $u_{1}$ and $u_{2}$ are of the order of $\epsilon$ which is small, then formally we have:

$$
P_{1}-P_{2} \approx N \log N u_{1} u_{2}\left(u_{2}-u_{1}\right) e^{2 l t}=O\left(\epsilon^{3}\right)
$$

That is, the difference between the two probabilities requires third-order terms in the mutation rate. However, this is misleading because the consecutive terms in this expansion are not necessarily small, as they involve the exponents $e^{l t}$, and for realistic parameter values they can be of order one.

\section{References}

Arkin, M.R., Wells, J.A. (2004). Small-molecule inhibitors of protein-protein interactions: progressing towards the dream. Nat. Rev. Drug. Discov. 3, 301-317.

Bailey, N. (1964). The elements of stochastic processes with applications to the natural sciences. New York: Wiley.

Bellomo, N., Chaplain, M., Angelis, E.D. (Eds.) (2008a). Selected topics on cancer modeling: genesisevolution-immune competition-therapy. Basel: Birkhäuser.

Bellomo, N., Li, N.K., Maini, P.K. (2008b). Math. Model Method Appl. Sci. 18, 593-646.

Bradeen, H.A., Eide, C.A., O’Hare, T., Johnson, K.J., Willis, S.G., Lee, F.Y., Druker, B.J., Deininger, M.W. (2006). Comparison of imatinib mesylate, dasatinib (BMS-354825), and nilotinib (AMN107) in an N-ethyl-N-nitrosourea (ENU)-based mutagenesis screen: high efficacy of drug combinations. Blood 108, 2332-2338.

Byrne, H.M., Alarcon, T., Owen, M.R., Webb, S.D., Maini, P.K. (2006). Modelling aspects of cancer dynamics: a review. Philos. Transact. A Math. Phys. Eng. Sci. 364, 1563-1578.

Coldman, A.J., Goldie, J.H. (1985). Role of mathematical modeling in protocol formulation in cancer chemotherapy. Cancer Treat. Rep. 69(10), 1041-1048.

Coldman, A.J., Goldie, J.H. (1986). A stochastic model for the origin and treatment of tumors containing drug-resistant cells. Bull. Math. Biol. 48(3-4), 279-292.

Colucci, G., Gebbia, V., Galetta, D., Riccardi, F., Cariello, S., Gebbia, N. (1997). Cisplatin and vinorelbine followed by ifosfamide plus epirubicin vs. the opposite sequence in advanced unresectable stage III and metastatic stage IV non-small-cell lung cancer: a prospective randomized study of the Southern Italy Oncology Group (GOIM). Br. J. Cancer 76, 1509-1517.

Day, R.S. (1986a). A branching-process model for heterogeneous cell populations. Math. Biosci. 78, 7390.

Day, R.S. (1986b). Treatment sequencing, asymmetry, and uncertainty: protocol strategies for combination chemotherapy. Cancer Res. 46, 3876-3885.

Deininger, M.W. (2007). Optimizing therapy of chronic myeloid leukemia. Exp. Hematol. 35, 144-154.

Deininger, M.W., Druker, B.J. (2003). Specific targeted therapy of chronic myelogenous leukemia with imatinib. Pharmacol. Rev. 55, 401-423.

Deisboeck, T.S., Zhang, L., Yoon, J., Costa, J. (2009). In silico cancer modeling: is it ready for prime time? Nat. Clin. Pract. Oncol. 6, 34-42.

Fulfaro, F., Valerio, M.R., Badalamenti, G., Gebbia, N., Russo, A. (2003). Antiblastic drug combinations with ifosfamide: an update. Oncology 65 Suppl. 2, 21-30.

Gaffney, E.A. (2005). The mathematical modelling of adjuvant chemotherapy scheduling: incorporating the effects of protocol rest phases and pharmacokinetics. Bull. Math. Biol. 67(3), 563-611.

Gardiner, C. (2004). Handbook of stochastic methods: for physics, chemistry and the natural sciences. Berlin: Springer. 
Gebbia, V., Galetta, D., Caruso, M., Verderame, F., Pezzella, G., Valdesi, M., Borsellino, N., Pandolfo, G., Durini, E., Rinaldi, M., Loizzi, M., Gebbia, N., Valenza, R., Tirrito, M.L., Varvara, F., Colucci, G. (2003). Gemcitabine and cisplatin versus vinorelbine and cisplatin versus ifosfamide + gemcitabine followed by vinorelbine and cisplatin versus vinorelbine and cisplatin followed by ifosfamide and gemcitabine in stage IIIB-IV non small cell lung carcinoma: a prospective randomized phase III trial of the Gruppo Oncologico Italia Meridionale. Lung Cancer 39, 179-189.

Goldie, J.H., Coldman, A.J. (1979). A mathematic model for relating the drug sensitivity of tumors to their spontaneous mutation rate. Cancer Treat. Rep. 63(11-12), 1727-1733.

Goldie, J.H., Coldman, A.J. (1983a). A model for resistance of tumor cells to cancer chemotherapeutic agents. Math. Biosci. 65, 291-307.

Goldie, J.H., Coldman, A.J. (1983b). Quantitative model for multiple levels of drug resistance in clinical tumors. Cancer Treat. Rep. 67(10), 923-931.

Goldie, J.H., Coldman, A.J. (1998). Drug resistance in cancer: mechanisms and models. Cambridge: Cambridge University Press.

Goldie, J.H., Coldman, A.J., Gudauskas, G.A. (1982). Rationale for the use of alternating non-crossresistant chemotherapy. Cancer Treat. Rep. 66, 439-449.

Grossi, F., Aita, M., Follador, A., Defferrari, C., Brianti, A., Sinaccio, G., Belvedere, O. (2007). Sequential, alternating, and maintenance/consolidation chemotherapy in advanced non-small cell lung cancer: a review of the literature. Oncologist 12, 451-464.

Katouli, A.A., Mathematical modeling of cancer drug treatment with cross-resistance. Ph.D. thesis UCI (Expected 2009).

Komarova, N.L. (2006). Stochastic modeling of drug resistance in cancer. J. Theor. Biol. 239, 351-366.

Komarova, N.L., Wodarz, D. (2005). Drug resistance in cancer: principles of emergence and prevention. Proc. Natl. Acad. Sci. USA 102, 9714-9719.

Komarova, N.L., Wu, L., Baldi, P. (2007). The fixed-size Luria-Delbruck model with a nonzero death rate. Math. Biosci. 210, 253-290.

Komarova, N.L., Katouli, A.A., Wodarz, D. (2009). Combination of two but not three current targeted drugs can improve therapy of chronic myeloid leukemia. PLOS ONE 4, e4423.

Norton, L., Day, R. (1985). Potential innovations in scheduling of cancer chemotherapy. In: Devita, V.T., Hellman, S., Rosenberg, S.A. (Eds.), Important advances in oncology (pp. 57-72). Philadelphia: Lippincott, Williams \& Wilkins.

Novozhilov, A.S., Karev, G.P., Koonin, E.V. (2006). Biological applications of the theory of birth-anddeath processes. Brief. Bioinform. 7, 70-85.

O’Dwyer, M.E., Mauro, M.J., Druker, B.J. (2002). Recent advancements in the treatment of chronic myelogenous leukemia. Annu. Rev. Med 53, 369-381.

Preziosi, L. (Ed.) (2003). Cancer modelling and simulation. London/Boca Raton: Chapman and Hall/CRC Press.

Quints-Cardama, A., Kantarjian, H., Cortes, J. (2007). Flying under the radar: the new wave of BCR-ABL inhibitors. Nat. Rev. Drug. Discov. 6, 834-848.

Sanga, S., Sinek, J.P., Frieboes, H.B., Ferrari, M., Fruehauf, J.P., Cristini, V. (2006). Mathematical modeling of cancer progression and response to chemotherapy. Expert Rev. Anticancer Ther. 6, 1361-1376.

Swierniak, A., Kimmel, M., Smieja, J. (2009). Mathematical modeling as a tool for planning anticancer therapy. Eur. J. Pharmacol. 625, 108-121.

Weisberg, E., Manley, P.W., Cowan-Jacob, S.W., Hochhaus, A., Griffin, J.D. (2007). Second generation inhibitors of BCR-ABL for the treatment of imatinib-resistant chronic myeloid leukemia. Nat. Rev. Cancer 7, 345-356.

Wodarz, D., Komarova, N.L. (2005). Computational biology of cancer: lecture notes and mathematical modeling. Singapore: World Scientific.

Zhang, J., Yang, P.L., Gray, N.S. (2009). Targeting cancer with small molecule kinase inhibitors. Nat. Rev. Cancer 9, 28-39. 